\title{
On the Universal Partition Theorem for 4-Polytopes
}

\author{
H. Günzel \\ Department of Mathematics-C, Aachen University of Technology, \\ D-52056 Aachen, Germany \\ harald@mathc.RWTH-Aachen.de
}

\begin{abstract}
By means of sign-patterns any finite family of polynomials induces a decomposition of $\mathbb{R}^{n}$ into basic semialgebraic sets. In case of integer coefficients the latter decomposition roughly appears to be a partition into realization spaces of 4-polytopes. The latter is stated by the Universal Partition Theorem for 4-polytopes by Richter-Gebert. The present paper presents a different proof. As its main tool, the von Staudt polytope is introduced. The von Staudt polytope constitutes the polytopal equivalent of the well-known von Staudt constructions for point configurations. With the aid of the von Staudt polytope the original ideas of universality theory can be directly applied to the polytopal case. Moreover, a new method for representing real values (on a computation line) by polytopal means is presented. This method implies a bundling strategy in order to duplicate the encoded information. Based on this approach, the following complexity result is obtained. The incidence code of a polytope, exhibiting a realization space equivalent to a given semialgebraic set, can be computed in the same time that it requires to generate the defining polynomial system.
\end{abstract}

\section{Introduction}

As a consequence of Mnëv's Universality Theorem for oriented matroids realization spaces of polytopes can be as "complex" as arbitrary basic semialgebraic sets (see [7], [2], [8], [11], and [4]). This stands in contrast to the well-known Steinitz Theorem (see [12] and [14]), which implies that realization spaces of 3-polytopes are always topologically trivial. The result for polytopes is obtained from the oriented matroid case via Gale diagrams (see [3]); however, it leaves open whether we would need an infinite number of polytope dimensions to represent all basic semialgebraic sets by realization spaces. The latter problem has been settled by Richter-Gebert [9], [10], who in fact has restricted the dimension to 4 . Due to a fundamental new idea, the latter proof can adapt the scheme of argumentation known from the case of oriented matroids.

The aforementioned scheme reflects the history of universality theory for geometrical 
objects, and has the following main ingredients. First, there is a projective scale providing a geometrical equivalent of the reals. Second, von Staudt constructions (see [6] and [13]) provide a computation of polynomials in a geometrical framework. The possible use of the latter tools in the area of universality theorems - for point configurations-has been independently discovered by Mnëv [7], and Bokowski and Sturmfels [2]. The work of Mnëv even includes the main ideas of how to settle the orientation problem arising by complex computations in the plane. Third, geometrical means of transmission of data decompose the computation into small units, which are easier to treat. Such techniques have been used by Shor [11] (Desargues configuration-for the binary case), and by Günzel et al. [4], [5] (perspectivities-for real-valued computation, which appears in the context of universality theorems). Fourth, the (Shor) normal form [11] provides a computation with estimates of the linear order of the intermediate results.

All these tools are intrinsically used in Richter-Gebert's proof. However, an application to the 4-polytope case only became possible through a new fundamental idea: define an auxiliary projective scale by polytopal tools! In [9] the slopes of a $2 k$-gon with parallel edges were used as an appropriate equivalent of the reals. Moreover, the possible application of perspectivities in a polytopal setting requires a specific locus, where the corresponding construction becomes "invisible" for the rest of the polytope. This leads to the connected sum operation introduced by Richter-Gebert. The latter operation glues two polytopes at a common facet. The 2-skeleton of the common facet has to imply its flatness in all realizations of the combined polytope. The reason for that restriction is to ensure the invertability of the connected sum operation.

Richter-Gebert then considers his encoding polygon and duplicates the encoded information by means of perspectivities on the boundary complex of a 4-polytope. The presence of the perspectivities is ensured by Lawrence extensions; see also the textbook by Ziegler [14]. Finally, the arithmetical operations ensuring the defining polynomials have to be fixed by polytopal tools. In [9] this is done by means of a specific (small) 4-polytope forcing a certain harmonic relation on the computation line. With a considerable effort of connected sum operations the harmonic polytope is then employed to fix the desired arithmetical operations, where four structurally different cases appear.

In this paper a different proof is presented. The basis thereof is formed by a new type of a normal form (similar to Shor's [11]), which represents the computation of the defining polynomials by affine isomorphisms between triples of points. Through this approach all the different arithmetical operations can be expressed in a unified way, implying the first considerable simplification of their polytopal representation. The computation line is then encoded by a polygon. Although this idea forms the main ingredient adapted from the original proof by Richter-Gebert, the encoding used differs substantially from the original. Here, the computation line is the affine hull of an edge of the encoding polygon, a fact providing duplication of the information without the use of perspectivities (at this step). In fact, the encoding polygons are bundled at the (common) computation line. This second simplification allows the computation of the incidence code of a 4-polytope, with a realization space equivalent to a given basic semialgebraic set, as fast as the system of polynomial functions defining the given set. Finally, the affine isomorphisms on the computation line are represented by pairs of perspectivities. This is in fact the fundamental idea behind von Staudt's constructions. The new aspect, here, is the direct application of the perspectivities to the encoding polygons, and not 
only to lines. In such a way the von Staudt polytope arises. The von Staudt polytope may be considered the main contribution of this paper. It directly converts the von Staudt constructions from an instrument for point configurations to a polytopal tool. In other words, the original ideas of universality theory - to consider a computation line and von Staudt constructions - have found their polytopal analog. Beside this, the use of only one polytopal building block forms a third simplification of the proof. No additional connected sum operations are required, except two Lawrence extensions, which fix the perspectivities used.

Remark 1. The method of a bundled product — see Section 3.4-was developed by the author before he knew about Richter-Gebert's work [9]. It originated with the intention to lift planar point configurations to 5-polytopes with equivalent realization spaces. In view of Mnëv's Universality Theorem for oriented matroids [7], this would provide a Universality Theorem for 5-polytopes. However, the author's investigations ran into some problems, which arose from the required calculation of the face lattice. RichterGebert's fundamental idea, to consider a polygonal equivalent to the reals instead of more complex configurations, also brought the breakthrough for the author's approach. The author then worked on a combination of both ideas, which resulted in this paper. Besides the aforementioned influence — in autumn 1994-both works on the universality theory for polytopes ran independently.

The paper is organized so that the reader comes step by step from the defining polynomials to more and more geometrical and polytopal representations thereof. In Section 2 the basic definitions are cited (and partially strengthened), and the Universal Partition Theorem is formulated. The entire proof is presented in Section 3. In Section 4 the reader finds a structural analysis of the face lattice of the von Staudt polytope, which is not necessary to follow the proof. The first step of the proof (Sections 3.1 and 3.2) represents the defining system of polynomials by a set of affine transformations between triples of points on the real line. In Section 3.3 the first polytopal tool is presented: the encoding polygon. Sufficiently many copies of the encoding polygon are bundled in Section 3.4 to form the starting polytope. The assertion on the computation of the incidence code in linear time, however, requires a smaller number of copies of the encoding polygon; compare the end of Section 3.9. A preview to the remaining (polytopal) steps of the proof is given in Section 3.5. In Section 3.6 one can see how the face lattice of the polytope formed from bundled polygons determines the order of the encoded numbers. The problems arising for the concept of stable equivalence, in connection with the connected sum operation, are discussed in Section 3.7. The construction of the von Staudt polytope is only presented in Section 3.8. For the first reading it is therefore suggested looking at Lemma 8 and Section 3.3 first, and then jump directly to Section 3.8. Finally, Section 3.9 assembles all the material.

\section{The Universal Partition Theorem}

The list of all faces of a polytope $P$, partially ordered by inclusion, forms a graded atomic and co-atomic lattice $\mathcal{L}(P)$. The minimal element of $\mathcal{L}(P)$ is $\emptyset$ and the maximal set is 
$P$. The vertices and facets are the atoms and co-atoms of the lattice, respectively. For a graded lattice we can define its dimension by means of maximal ascending sequences. Let $\mathcal{L} \mathcal{A} \mathcal{T}(d, k)$ denote the set of graded co-atomic lattices with dimension $d$ and $k$ vertices. Note that the lattice is already determined by the set of incidences between the atoms and the co-atoms, which is referred to as the incidence code.

Intuitively, any polytope $P$ with $\mathcal{L}(P)=\mathcal{L}$ could be regarded as a realization of the lattice $\mathcal{L}$. However, in order to obtain a realization space with a simpler topology, we use a factorization up to affine isomorphisms. To this end, we consider vertices (of our lattice) such that the corresponding vertices of a realization (a polytope) are necessarily affinely independent: For $\mathcal{L} \in \mathcal{L} \mathcal{A T}(d, k)$ a set of vertices $p_{1}, \ldots, p_{d+1}$ is called a basis if for any $i=1, \ldots, d$ there exists a face $F \in \mathcal{L}$ containing $p_{1}, \ldots, p_{i}$, but not $p_{i+1}, \ldots, p_{d+1}$. (Note that any graded lattice has a basis.) Now let $\mathcal{L} \mathcal{A} \mathcal{T}^{*}(d, k)$ be the set of those lattices from $\mathcal{L} \mathcal{A} \mathcal{T}(d, k)$ for which $p_{1}, \ldots, p_{d+1}$ forms a basis. In the following, we identify a polytope $P \subset \mathbb{R}^{d}$ with the vector of its vertices $\left(p_{1}, \ldots, p_{k}\right) \in \mathbb{R}^{d k}$.

Definition 2. Let $\mathcal{L} \in \mathcal{L} \mathcal{A} \mathcal{T}^{*}(d, k)$. Then a polytope $P$ in $\mathbb{R}^{d}$ is called a realization of $\mathcal{L}$ if $\mathcal{L}(P)=\mathcal{L}$ and, moreover, the first vertices $p_{1}, \ldots, p_{d+1}$ coincide with the affine basis $p_{1}=0, p_{2}=(1,0, \ldots, 0), \ldots$, and $p_{d+1}=(0, \ldots, 0,1)$. The set of all realizations of $\mathcal{L}$ forms a subspace of $\mathbb{R}^{d k}$. It is called the realization space of $\mathcal{L}$, and is denoted by $\mathcal{R}(\mathcal{L})$.

The realization spaces of 4-polytopes are compared with basic semialgebraic sets defined over $\mathbb{Z}$. Such sets are defined to be the finite intersection of sets of the form $\left\{x \in \mathbb{R}^{n} \mid g(x)=0\right\}$, and $\left\{x \in \mathbb{R}^{n} \mid g(x)>0\right\}$, where $g$ stands for a polynomial with integer coefficients. For more details on (basic) semialgebraic sets we refer to [1]. The polynomials $g \in \mathbb{Z}[X]$ can be computed by straight line programs, which manipulate the coefficients of already computed polynomials in order to compute new polynomials.

Definition 3. A straight line program on $n$ variables is a sequence of polynomials $\mathcal{F}=\left\{f_{0}, \ldots, f_{\alpha}\right\}$ together with mappings

$$
\begin{aligned}
& \circ:\{n+1, \ldots, \alpha\} \rightarrow\{+,-, \times\}, \quad \beta \mapsto \circ_{\beta} \quad \text { (operations), } \\
& i, j:\{n+1, \ldots, \alpha\} \rightarrow\{0, \ldots, \alpha-1\} \quad \text { (predecessors) }
\end{aligned}
$$

satisfying the following conditions:

(i) $f_{0}=1, f_{1}=X_{1}, \ldots, f_{n}=X_{n}$,

(ii) $i(\beta), j(\beta)<\beta$,

(iii) $f_{\beta}=f_{i(\beta)} \circ_{\beta} f_{j(\beta)}$.

The number $\alpha$ is called the length of the straight line program $(\mathcal{F}, \circ, i, j)$.

Now we describe the type of mappings that is going to be used to compare the structure of realization spaces with basic semialgebraic sets. Here, we follow the concept of stable equivalence introduced by Mnëv [7], [8], we especially refer to the refined concept introduced by Richter-Gebert [9], which is slightly strengthened here again. Compared with [9] our setting immediately implies that a stable projection mapping is a trivial fibration. 
Definition 4 (Stable Projection). Let $\Sigma$ denote a (finite) index set such that $\mathcal{V}=$ $\left\{V_{\sigma} \mid \sigma \in \Sigma\right\}$ and $\mathcal{W}=\left\{W_{\sigma} \mid \sigma \in \Sigma\right\}$ are families of pairwise disjoint subsets of $\mathbb{R}^{n}$ and $\mathbb{R}^{n+d}$, respectively. Put $V:=\bigcup_{\sigma} V_{\sigma}$ and $W:=\bigcup_{\sigma} W_{\sigma}$. Let $\Pi: \mathbb{R}^{n+d} \rightarrow \mathbb{R}^{n}$ denote the canonical projection deleting the last $d$ coordinates. We say that $\mathcal{V}$ is a stable projection of $\mathcal{W}$ if the following conditions are satisfied:

(i) $\Pi\left(W_{\sigma}\right)=V_{\sigma}, \sigma \in \Sigma$,

(ii) the $n$-parametric family $M(v) \subset \mathbb{R}^{d}, v \in \mathbb{R}^{n}$, defined by setting

$$
M(v):=\left\{w \in \mathbb{R}^{d} \mid(v, w) \in W\right\},
$$

consists of interiors of polyhedra,

(iii) $M(v)$ can be described by means of a finite set of linear inequality constraints

$$
M(v)=\left\{w \in \mathbb{R}^{d} \mid \varphi_{i}(v) * w>\psi_{i}(v), i \in I\right\},
$$

such that the coordinate functions $\varphi_{i, j}, \psi_{i}: \mathbb{R}^{n} \rightarrow \mathbb{R}$ are polynomial functions with integer coefficients. The symbol " $*$ " denotes the scalar product.

The mapping $\Pi$ is referred to as a stable projection (mapping).

Definition 5 (Stable Equivalence). Two families $\mathcal{V}$ and $\mathcal{W}$ as in Definition 4 are called rationally equivalent if there exists a homeomorphism $\varphi: V \rightarrow W$ such that $\varphi\left(V_{\sigma}\right)=$ $W_{\sigma}$, and both functions $\varphi$ and $\varphi^{-1}$ are rational functions (with integer coefficients).

Two families $\mathcal{V}$ and $\mathcal{W}$ are stably equivalent (abbreviated by $\mathcal{V} \sim \mathcal{W}$ ) if they belong to the same equivalence class generated by stable projections and rational equivalence. A finite composition of stable projections and rational equivalences is called a stable composition.

Main Theorem (Universal Partition Theorem for $(1, \infty)^{n}$-Partitions). $\operatorname{Let}(\mathcal{F}, \circ, i, j)$ be a straight line program, and let $g_{1}, \ldots, g_{m} \in \mathcal{F}$. The polynomials $g_{1}, \ldots, g_{m} \in \mathbb{Z}[X]$ induce a partition $\mathcal{S}:=\left\{S_{\sigma} \mid \sigma \in\{-1,0,1\}^{m}\right\}$ of $(1, \infty)^{n}$ into basic semialgebraic sets, where $S_{\sigma}:=\left\{x \in(1, \infty)^{n} \mid \operatorname{sign} g_{i}(x)=\sigma_{i}, i=1, \ldots, m\right\}$.

(i) Then there exist lattices $\mathcal{L}_{\sigma} \in \mathcal{L} \mathcal{A} \mathcal{T}^{*}(4, k)$ such that

$$
\mathcal{S} \sim \mathcal{R}
$$

where $\mathcal{R}$ denotes the family of realization spaces $\mathcal{R}:=\left\{\mathcal{R}\left(\mathcal{L}_{\sigma}\right) \mid \sigma \in \Sigma\right\}$, which are defined by $\left\{\mathcal{L}_{\sigma}\right\}_{\sigma}$. (Actually, a stable composition exists.)

(ii) The face lattice $\mathcal{L}_{\sigma}$ can be computed in quadratic time (the incidence code even in linear time), both with respect to the length of the straight line program.

Corollary 6 (Universal Partition Theorem). The assertion of the Main Theorem also holds for partitions of $\mathbb{R}^{n}$ instead of $(1, \infty)^{n}$. Both assertions are in fact equivalent; including the complexity result.

Proof. In order to see that the original assertion implies the assertion for partitions of the entire space, we substitute the variable $x_{i} \in \mathbb{R}$ by a pair $\left(x_{i}^{+}, x_{i}^{-}\right) \in(1, \infty)$ such that 
$x_{i}=x_{i}^{+}-x_{i}^{-}$. A moment's reflection shows that $\left(x^{+}, x^{-}\right) \mapsto\left(x^{+}, x^{-}, x\right) \mapsto x$ forms a stable composition; see also [4] and [9]. The other direction is obvious.

\section{Proof of the Theorem}

\subsection{Nonnegative Coefficients}

The functions $f_{0}, \ldots, f_{\alpha}$ of our straight line program are replaced by pairs of polynomials $\left(f_{\beta}^{+}, f_{\beta}^{-}\right)$with nonnegative coefficients, such that we have $f_{\beta}=f_{\beta}^{+}-f_{\beta}^{-}$. To this end let $1=1-0$ (i.e., $1^{+}=1,1^{-}=0$ ), and $X_{i}=X_{i}-0$. If $\circ_{\beta}=+$ we use $f_{\beta}^{+}=f_{i(\beta)}^{+}+f_{j(\beta)}^{+}$ and $f_{\beta}^{-}=f_{i(\beta)}^{-}+f_{j(\beta)}^{-}$. For $\circ_{\beta}=-$ we put $f_{\beta}^{+}=f_{i(\beta)}^{+}+f_{j(\beta)}^{-}$and $f_{\beta}^{-}=f_{i(\beta)}^{-}+f_{j(\beta)}^{+}$, and for $\circ_{\beta}=\times$ we put $f_{\beta}^{+}=f_{i(\beta)}^{+} f_{j(\beta)}^{+}+f_{i(\beta)}^{-} f_{j(\beta)}^{-}$and $f_{\beta}^{-}=f_{i(\beta)}^{+} f_{j(\beta)}^{-}+f_{i(\beta)}^{-} f_{j(\beta)}^{+}$.

In such a way we obtain a straight line program $\mathcal{F}^{\prime}$ of length $\gamma<3 \alpha$ which excludes the operation $o_{\beta} \neq-$, and which computes the defining pairs of polynomials $\left(g_{1}^{+}, g_{1}^{-}\right), \ldots,\left(g_{m}^{+}, g_{m}^{-}\right)$. For simplicity delete all additions and multiplications with the zero function and multiplications with 1 from the new straight line program $\left(\mathcal{F}^{\prime}, \circ, i, j\right)$. Put $f_{n+1}:=2$, i.e., $\circ(n+1)=+, i, j(n+1)=1$. Finally, we can easily ensure (by some extra addition on both sides) that none of the defining polynomials $g_{1}^{+}, g_{1}^{-}, \ldots, g_{m}^{+}, g_{m}^{-}$ vanishes; this might increase the length of the straight line program to some $\gamma<6 \alpha$.

\subsection{Computation on a Line}

In this section the operations of the straight line program are encoded by affine transformations between triples of points on the real line. This method is novel and enables us to treat the computational procedure in a unified way. It extends the concept of a Shor normal form [9], [11] in such a way that only one type of an encoding relation is required. In the work of Richter-Gebert [9], four different cases (caused by different operations of the computational procedure) had to be distinguished. The construction of the normal form roughly works as follows. In Section 3.1 the original straight line program has been replaced by a new one only using the operations of addition and multiplication, thus all the intermediate results stay within the interval $(1, \infty)$. The appearing values of polynomial evaluation are not required to be completely ordered; in particular the input variables can have arbitrary order. The idea of a normal form consists of the fact that the order of some particular results of polynomial evaluation is predetermined by the straight line program, thus the polynomials themselves and their domain. For example, the value of $f_{i}+f_{j}$ must be greater than $f_{i}, f_{j}, 1$ provided that $f_{i}, f_{j}>1$. What we do is to chose small sets of polynomials with predefined order, such as $0<1<f_{i}<f_{j}$. The precise value of $f_{j}$ is unknown; nevertheless it must be finite. We have the freedom to chose any ascenting affine isomorphism ( $\operatorname{say} \varphi: \mathbb{R} \rightarrow \mathbb{R}$ ) to encode the values of $f_{i}, f_{j}$ on the image line; they are given by the position of the images of $0,1, f_{i}$ and $0,1, f_{j}$, respectively. Having encoded the first set of polynomials into what we call a block there still remains the interval $\left(\varphi\left(f_{j}\right), \infty\right)$ for an encoding of the next ordered set of polynomials. This inductive procedure yields an encoding of all the intermediate 
function values in a linearly ordered way; in fact the fixed order type does not imply any further partitioning of the given basic semialgebraic sets. The next step consists in the encoding of the functional dependence between the different intermediate results, which is given by the straight line program. To this end our blocks must have been chosen appropriately. By means of some new function evaluations which have not been present so far in the straight line program it is possible to ensure all the functional dependences (and order properties) by means of affine isomorphisms between triples of points from different blocks. After this introduction we start with the formal part.

Consider a $k$-tuple $q=\left(q_{1}, \ldots, q_{k}\right)$ on the real line $\mathbb{R}$. It is called ordered if $0<$ $q_{1}<\cdots<q_{k}$. We compare ordered triples, where we always assume them to belong to an ordered $k$-tuple $q$ with $k \geq 6$. Two triples $\left(q_{i_{1}}, q_{i_{2}}, q_{i_{3}}\right)$ and $\left(q_{j_{1}}, q_{j_{2}}, q_{j_{3}}\right)$ are called comparable if they are ordered and their convex hulls $\left[q_{i_{1}}, q_{i_{3}}\right]$ and $\left[q_{j_{1}}, q_{j_{3}}\right]$ are disjoint. Let $A$ denote the affine isomorphism sending $q_{i_{1}}$ to $q_{j_{1}}$ and $q_{i_{2}}$ to $q_{j_{2}}$. We define the sign of a pair of triples as follows:

$$
\operatorname{sign}\left(\left(q_{i_{1}}, q_{i_{2}}, q_{i_{3}}\right),\left(q_{j_{1}}, q_{j_{2}}, q_{j_{3}}\right)\right):= \begin{cases}-1, & A\left(q_{i_{3}}\right)<q_{j_{3}}, \\ 0, & A\left(q_{i_{3}}\right)=q_{j_{3}}, \\ +1, & A\left(q_{i_{3}}\right)>q_{j_{3}} .\end{cases}
$$

The sign-function compares triples belonging to clusters. Here, a cluster is a subset of the $k$-tuple that is equipped with its own affine scale, measuring the relative position of the points involved. The points of the cluster stand for specific function values, which are actually encoded that way. To define the scale there are points representing the numbers 0 and 1. Having this in mind, the sign-function can be used in a twofold way. The function values represented by $q_{i_{3}}$ and $q_{j_{3}}$ are compared if both $q_{i_{1}}$ and $q_{j_{1}}$ stand for 0 , and both $q_{i_{2}}$ and $q_{j_{2}}$ for 1 . Otherwise, some computation is encoded. To be sure of the precise result, only the sign-value 0 is allowed in such a case. The latter situation calls for its own name: the $k$-tuple $q$ is said to satisfy the condition $C:\left(q_{i_{1}}, q_{i_{2}}, q_{i_{3}}\right) \approx\left(q_{j_{1}}, q_{j_{2}}, q_{j_{3}}\right)$ if $\operatorname{sign}\left(\left(q_{i_{1}}, q_{i_{2}}, q_{i_{3}}\right),\left(q_{j_{1}}, q_{j_{2}}, q_{j_{3}}\right)\right)=0$. To define the realization space of ordered $k$-tuples an affine basis is fixed. The basis is given by the position of 0 and of another point, say $q_{1}=1$. The realization space of ordered $k$-tuples defined that way is denoted by $\mathbb{R}_{\text {ordered }}^{k}$. In the next step $\mathbb{R}_{\text {ordered }}^{k}$ is partitioned into smaller realization spaces which are associated with conditions and sign-mappings.

Definition 7. Let $\mathcal{C}=\left\{C_{1}, \ldots, C_{r}\right\}$ be a set of conditions on $\left\{q_{1}, \ldots, q_{k}\right\}$. Then the space

$$
\mathcal{R}_{\mathcal{C}}:=\left\{q \in \mathbb{R}_{\text {ordered }}^{k} \mid q \text { satisfies } C_{1}, \ldots, C_{r}\right\}
$$

is called the realization space of $\mathcal{C}$. We need a decomposition into smaller realization spaces. To this end consider a vector of sign-functions sign: $\mathbb{R}_{\text {ordered }}^{k} \rightarrow\{-1,0,+1\}^{m}$ on comparable pairs of triples. Let a sign-vector $\sigma \in\{-1,0,+1\}^{m}$ be given. Then the space

$$
\mathcal{R}_{\mathcal{C}}(\sigma):=\left\{q \in \mathcal{R}_{\mathcal{C}} \mid \operatorname{sign}(q)=\sigma\right\}
$$

is called the common realization space of $\mathcal{C}$ and $\sigma$. Let $\mathcal{R}(\mathcal{C}$, sign) denote the partition of $\mathcal{R}_{\mathcal{C}}$ into common realization spaces defined by setting $\mathcal{R}(\mathcal{C}$, sign $):=\left\{\mathcal{R}_{\mathcal{C}}(\sigma) \mid \sigma \in\right.$ $\left.\{-1,0,+1\}^{m}\right\}$. 


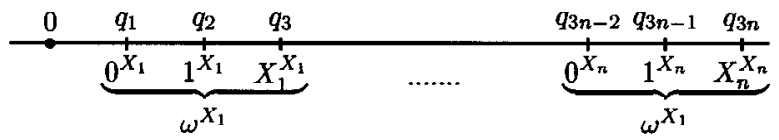

Fig. 1. Functions $X_{1}, \ldots, X_{n}$.

Lemma 8 (Normal Form). Let $\mathcal{S}$ denote the partition of $(1, \infty)^{n}$ into basic semialgebraic sets, as in the Main Theorem. There exist a set of conditions $\mathcal{C}=\left\{C_{1}, \ldots, C_{r}\right\}$ (on ordered $k$-tuples) and a vector of sign-functions sign: $\mathbb{R}_{\text {ordered }}^{k} \rightarrow\{-1,0,+1\}^{m}$ such that $\mathcal{S} \sim \mathcal{R}(\mathcal{C}$, sign $)$. The set of conditions $\mathcal{C}$ and the vector of sign-functions sign can be computed in linear time with respect to the length $\alpha$ of the straight line program $\mathcal{F}$. In particular, the number $k$ of points forming the $k$-tuple can be estimated by $k<11 \gamma<66 \alpha$ and the number $r$ of conditions by $r<7 \gamma<42 \alpha$.

Proof. We prove the lemma by induction over the length $\gamma$ of the straight line program $\left(\mathcal{F}^{\prime}, \circ, i, j\right)$ constructed in Section 3.1; note that $\gamma \geq n+1$. The appropriate shorter program is obtained by deleting the last operations of the original. We show the existence of a set of conditions $\mathcal{C}$ such that the following conditions hold (with appropriate mappings $q \mapsto x(q)$ and $\left.q \mapsto f_{i}^{\text {geom }}(q)\right)$ :

(i) $q \mapsto x(q)$ is a stable composition $\mathcal{R}_{\mathcal{C}} \rightarrow(1, \infty)^{n}$,

(ii) $f_{i}(x(q))=f_{i}^{\text {geom }}(q), i=1, \ldots, r$;

the mappings $x(q)$ and $f_{i}^{\text {geom }}(q)$ are defined below.

The first step deals with the smallest case, namely $\gamma=n+1, \mathcal{F}^{\prime}=\left\{1, X_{1}, \ldots, X_{n}\right\}$. Put $k:=3 n$ and $r:=0$. Consider an arbitrary ordered $3 n$-tuple $q=\left(q_{1}, \ldots, q_{3 n}\right) \in$ $\mathbb{R}_{\text {ordered }}^{3 n}$. To symbolize their meaning, the points $q_{1}, \ldots, q_{3 n}$ are endowed with new labels $0^{f}, 1^{f}, f^{f}$, for $f=X_{1}, \ldots, X_{n}$, as shown in Fig. 1 . For $f=X_{1}, \ldots, X_{n}$ we define an affine scale $\omega^{f}: \mathbb{R} \rightarrow \mathbb{R}$ to be the affine isomorphism sending $0^{f}, 1^{f}$ to 0 and 1 , respectively. Then $f^{\text {geom }}(q):=\omega^{f}\left(f^{f}\right)$ is the geometrical function evaluation of $f$ for the given $k$-tuple. In particular, this defines $x^{\text {geom }}(q):=\left(X_{1}^{\text {geom }}, \ldots, X_{n}^{\text {geom }}\right)(q)$. Property (ii) says that the geometrical function evaluation $q \mapsto f_{i}^{\text {geom }}(q)$ coincides with the arithmetical evaluation given by $q \mapsto x(q) \mapsto f_{i}(x(q))$. In the first step property (ii) holds by setting $x(q):=x^{\text {geom }}(q)$. It remains to verify (i). It is clear that $q \mapsto\left(q, X_{1}(q), \ldots, X_{n}(q)\right)$ is a rational equivalence. Moreover, each mapping of forgetting the last point of $q$, namely $\left(q_{1}, \ldots, q_{l}, X_{1}(q), \ldots, X_{n}(q)\right) \mapsto\left(q_{1}, \ldots, q_{l-1}, X_{1}(q), \ldots, X_{n}(q)\right)$ is a rational equivalence (if $q_{l}$ is some $f^{f}$ or $l=1$ ) or a stable projection (otherwise). The composition of the latter mappings coincides with $q \mapsto x(q), \mathcal{R}_{\emptyset} \rightarrow$ $(1, \infty)^{n}$.

In the second step $(\gamma=n+2)$ we represent the equation $f_{n+2}=2=1+1$ by means of affine isomorphisms on $\mathbb{R}$. Consider an ordered $(k+9)$-tuple, which is an extension of the $k$-tuple used in the previous step. Recall that the $k$-tuple is a common realization of $(\mathcal{C}, \emptyset)$. The additional points are labeled as shown in Fig. 2. New affine scales $\omega^{*}, \omega^{+}: \mathbb{R} \rightarrow \mathbb{R}$ are defined analogously to step 1, i.e., $\omega^{*}\left(0^{*}\right)=0, \omega^{*}\left(1^{*}\right)=1$, 


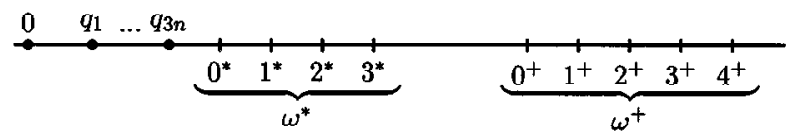

Fig. 2. Functions 2, 3.

etc. We use the following conditions:

$$
\begin{array}{ll}
C_{1}: & (0,1,2)^{*} \approx(0,1,2)^{+}, \\
C_{2}: & (0,1,3)^{*} \approx(0,1,3)^{+}, \\
C_{3}: & (0,1,2)^{*} \approx(1,2,3)^{+}, \\
C_{4}: & (0,1,3)^{*} \approx(1,2,4)^{+}, \\
C_{5}: & (0,1,2)^{*} \approx(0,2,4)^{+}
\end{array}
$$

where the upper indices behind the brackets represent upper indices of the coefficients inside, i.e., $(0,1,2)^{*}$ in $C_{1}$ abbreviates $\left(0^{*}, 1^{*}, 2^{*}\right)$. Condition $C_{1}$ does not guarantee the desired equation $\omega^{*}\left(2^{*}\right)=2$. In fact, $C_{1}$ stands for an affine isomorphism, i.e., it just means $\omega^{*}\left(2^{*}\right)=\omega^{+}\left(2^{+}\right)=$: $a$. Satisfying all the conditions $C_{1}, \ldots, C_{5}$, however, is equivalent to $a=2, b=3$, and $c=4$. This can be seen as follows:

$$
\begin{aligned}
C_{2} & \Leftrightarrow \omega^{*}\left(3^{*}\right)=\omega^{+}\left(3^{+}\right)=: b, \\
C_{3} & \Leftrightarrow \frac{a-0}{1-0}=\frac{b-1}{a-1}, \\
C_{4} & \Leftrightarrow \frac{b-0}{1-0}=\frac{c-1}{a-1}, \quad \text { where } c:=\omega^{+}\left(4^{+}\right), \\
C_{5} & \Leftrightarrow \frac{a-0}{1-0}=\frac{c-0}{a-0} .
\end{aligned}
$$

Finally, the numbers $k$ and $r$ are increased by 9 and 5, respectively. For function $2 \in \mathcal{F}^{\prime}$ we use the affine scale $\omega^{2}:=\omega^{*}$, i.e., $0^{2}:=0^{*}, 1^{2}:=1^{*}, 2^{2}:=2^{*}$. This implies property (ii). In view of property (i) it suffices to show that the mapping of forgetting the new points is a stable composition from the new realization space to the realization space of step 1 . The arguments must be chosen with some care. Indeed, deleting one single point destroys the encoded structure, therefore we have to delete all the new points nearly simultaneously. The position of the 7-tuple of new points is an affine mapping of the positions of the quadruple $\left(0^{*}, 3^{*}, 0^{+}, 1^{+}\right)$. Deleting all the other points (at once) yields a rational equivalence. The remaining points can be deleted step by step starting with the point exhibiting the largest $q$-index, yielding stable projections.

In the third step of our proof we assume that the lemma already applies to straight line programs of length $\gamma-1 \geq n+2$ (just delete the last operation of the original one). We add new points and new conditions in order to encode the computation of $f_{\gamma}$.

Case 1. Multiplication to be encoded: $\circ_{\gamma}=\times$. We add four points according to Fig. 3 and two conditions, i.e., $k$ is increased by 4 and $r$ by 2 :

$$
\begin{array}{ll}
\left(0,1, f_{i}\right)^{f_{\gamma}} \approx\left(0,1, f_{i}\right)^{f_{i}} & \text { meaning } \quad \omega^{f_{\gamma}}\left(f_{i}^{f_{\gamma}}\right)=f_{i}(x), \\
\left(0, f_{i}, f_{\gamma}\right)^{f_{\gamma}} \approx\left(0,1, f_{j}\right)^{f_{j}} & \text { saying } \quad \omega^{f_{\gamma}}\left(f_{\gamma}^{f_{\gamma}}\right)=f_{\gamma}(x) .
\end{array}
$$




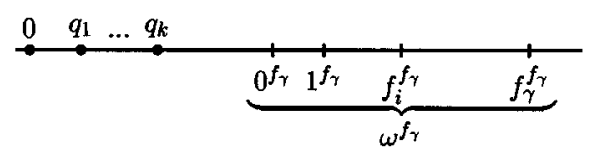

Fig. 3. Operation $x$.

Case 2. Addition to be encoded: $\circ_{\gamma}=+$. We can assume that $f_{i} \neq 1$; recall step 1 . New points are added according to Fig. 4 (in case $f_{j}=1$ not all of them). Here are the corresponding conditions:

$$
\begin{aligned}
\left(0,1, f_{i}\right)^{*} & \approx\left(0,1, f_{i}\right)^{f_{i}}, \\
\left(1, f_{i}, 2 f_{i}-1\right)^{*} & \approx(0,1,2)^{2}, \\
\left(0, f_{i}, 2 f_{i}\right)^{*} & \approx(0,1,2)^{2}, \\
\left(2 f_{i}-1,2 f_{i}, 2 f_{i}+2\right)^{*} & \approx(0,1,3)^{2}, \\
\left(0,1, f_{i}\right)^{+} & \approx\left(0,1, f_{i}\right)^{*} \\
\left(0, f_{i}, f_{i}+1\right)^{+} & \approx\left(0,2 f_{i}, 2 f_{i}+2\right)^{*}, \\
\left(f_{i}, f_{i}+1, f_{\gamma}\right)^{+} & \approx\left(0,1, f_{j}\right)^{f_{j}} .
\end{aligned}
$$

Provided the earlier conditions are satisfied the above conditions are equivalent to the following assertions: $\omega^{*}\left(f_{i}^{*}\right)=f_{i}(x), \omega^{*}\left(\left(2 f_{i}-1\right)^{*}\right)=2 f_{i}(x)-1, \omega^{*}\left(\left(2 f_{i}\right)^{*}\right)=$ $2 f_{i}(x), \omega^{*}\left(\left(2 f_{i}+2\right)^{*}\right)=2 f_{i}(x)+2, \omega^{+}\left(f_{i}^{+}\right)=f_{i}(x), \omega^{+}\left(\left(f_{i}+1\right)^{+}\right)=f_{i}(x)+1$, and $\omega^{+}\left(f_{\gamma}^{+}\right)=f_{\gamma}(x)$.

In case $f_{j}=1$ the last point $f_{\gamma}^{+}$and the last condition are not added (instead, we take $\left.f_{\gamma}^{+}:=\left(f_{i}+1\right)^{+}\right)$. One easily checks that the involved points are always ordered in the described way. Finally, $k$ and $r$ are updated and $\omega^{f_{\gamma}}:=\omega^{+}$is chosen as the affine scale associated with $f_{\gamma}$.

Cases 1 and 2 . For any operation we add at most 11 points and 7 conditions. The mapping of forgetting the new points forms a stable composition. This can be seen analogously to step 2 .

In the fourth (and final) step of the proof we consider a realization space $\mathcal{R}_{\mathcal{C}}$ having the properties (i) and (ii). In view of these properties it suffices to compare the values of $\omega^{g_{i}^{+}}\left(g_{i}^{+}\right)$and $\omega^{g_{i}^{-}}\left(g_{i}^{-}\right)$by means of additional sign-functions; neither new points nor new conditions are required. We add the sign-functions

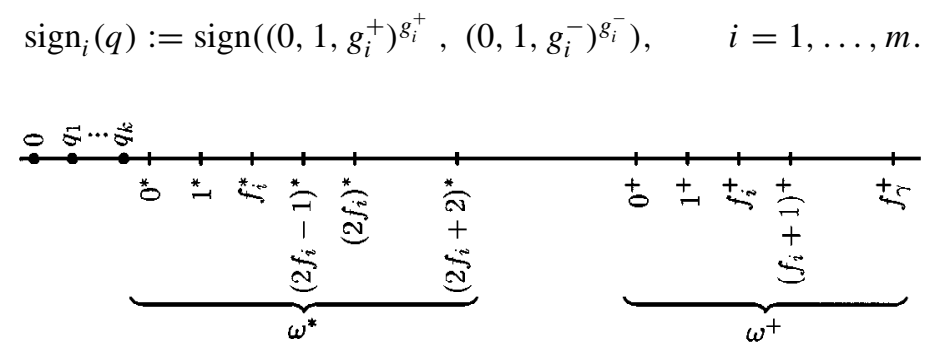

Fig. 4. Operation + . 
Then, in view of property (ii), it follows that

$$
\operatorname{sign}_{i}(q)=\operatorname{sign}\left(g_{i}^{+}(x(q))-g_{i}^{-}(x(q))\right) .
$$

Therefore the following holds for any sign-vector $\sigma \in\{-1,0,+1\}^{m}$ and any $q \in \mathcal{R}_{\mathcal{C}}$ :

$$
x(q) \in \mathcal{S}(\sigma) \quad \Leftrightarrow \quad q \in \mathcal{R}_{\mathcal{C}}(\sigma) .
$$

This completes the proof in view of property (i), showing the stable equivalence of the partitions of $\mathbb{R}^{n}$ and $\mathcal{R}_{\mathcal{C}}$ that are compared.

\subsection{The Encoding Polygon}

We define a (reduced) realization space of the $(k+3)$-gons. The $(k+3)$-gon $P \subset \mathbb{R}^{2}$ is identified with the vector $P=\left(p_{\infty}, p_{0}, p_{1}, \ldots, p_{k+1}\right)$ of its vertices, which are supposed to appear in the described order. The realization space $\mathcal{P}^{k+3}$ of the $(k+3)$ gons is defined in the same way as the realization space of a polytope: by fixing an affine basis. We choose the affine basis $p_{\infty}=(0,0), p_{0}=(1,0), p_{k+1}=(0,1)$. The reduced space of $(k+3)$-gons is then defined by setting

$$
\mathcal{P}_{\text {reduced }}^{k+3}:=\left\{\begin{array}{l|l}
P \in\left(\mathbb{R}^{2}\right)^{k+3} & \begin{array}{l}
p_{\infty}=(0,0), p_{0}=(1,0), p_{k+1}=(0,1), \\
a=(10,0), b=(0,10), \text { where } \\
a:=\operatorname{proj}\left(p_{k}, p_{k+1}\right) \cap \operatorname{proj}\left(p_{\infty}, p_{0}\right), \\
b:=\operatorname{proj}\left(p_{0}, p_{1}\right) \cap \operatorname{proj}\left(p_{\infty}, p_{k+1}\right)
\end{array}
\end{array}\right\} .
$$

Here, $\operatorname{proj}\left(p_{i}, p_{j}\right)$ stands for the projective line-in the projective closure $\mathbb{P}^{2}$ of $\mathbb{R}^{2}$ which connects the points $p_{i}$ and $p_{j}$. A moment's reflection shows that $\mathcal{P}_{\text {reduced }}^{k+3}$ represents the space of projective equivalence classes of $(k+3)$-gons. (For a verification choose the points $p_{\infty}, a, b, \operatorname{proj}\left(a, p_{k+1}\right) \cap \operatorname{proj}\left(b, p_{0}\right)$ as a projective basis of $\mathbb{P}^{2}$. Note that the constant 10 in the definition of $\mathcal{P}_{\text {reduced }}^{k+3}$ is arbitrary. In fact we only have to fix some constant in order to define a projective basis.) The fact that $\mathcal{P}_{\text {reduced }}^{k+3}$ represents the space of projective equivalence classes is used in the proof of Lemma 9 in order to show that the decoding mapping $\xi$ is a stable composition:

$$
\begin{aligned}
& \xi: \mathcal{P}^{k+3} \rightarrow \mathbb{R}_{\text {ordered }}^{k}, \\
& \xi: P \mapsto q .
\end{aligned}
$$

Before defining mapping $\xi$ recall that $\mathbb{R}_{\text {ordered }}^{k}$ is related to a specific affine basis of $\mathbb{R}$. In fact we have used the points 0 and $q_{1}=1$. The choice of this basis supported the proof of Lemma 8. In the present situation the affine basis 0 and $q_{k}=10$ is more appropriate. The transformation between both kinds of realizations of ordered $k$-tuples is obtained by affine isomorphisms. Since this yields a rational equivalence we do not distinguish between both representations of $\mathbb{R}_{\text {ordered }}^{k}$, however, in what follows we refer to the affine basis given by 0 and $q_{k}=10$.

The coefficient $q_{i}$ of $q$ is defined as the (nonstandard) coordinate of $\tilde{q}_{i}:=\operatorname{proj}\left(p_{\infty}, p_{0}\right)$ $\cap \operatorname{proj}\left(p_{i}, p_{i+1}\right)$ with respect to the projective basis $\left\{p_{\infty}, p_{0}, \tilde{q}_{k}\right\}$. The latter projective coordinate is defined to be the image of $\tilde{q}_{i}$ under the projective isomorphism 


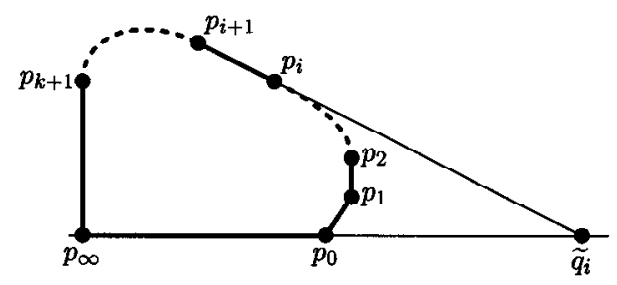

Fig. 5. The encoding polygon.

$\operatorname{proj}\left(p_{\infty}, p_{0}\right) \rightarrow \mathbb{P}=\overline{\mathbb{R}}$ sending $p_{\infty}, p_{0}, \tilde{q}_{k}$ to $\infty, 0,10$, respectively. For an illustration of this important definition see Fig. 5. Roughly speaking, the mapping $\xi$ enables us to convert sign-conditions on ordered $k$-tuples into shape-conditions of polygons. In view of (the proof of) Lemma 8, these shape-conditions even encode the given straight line program. For convenience we identify the encoded point $q_{i}:=\xi_{i}(P)$ with the intersection point $\widetilde{q}_{i}$. However, here, in view of stable composition, it is important to treat the images on the real line.

Lemma 9. The decoding mapping $\xi: \mathcal{P}^{k+3} \rightarrow \mathbb{R}_{\text {ordered }}^{k}$ is a stable composition.

Proof. The proof consists of two steps. In Step 2 we show that $\xi: \mathcal{P}_{\text {reduced }}^{k+3} \rightarrow \mathbb{R}_{\text {ordered }}^{k}$ is a stable composition. Step 1 is a reduction step. Here, we use the projective isomorphisms $\psi$ sending any $(k+3)$-gon to its projective equivalent in $\mathcal{P}_{\text {reduced }}^{k+3}$. Applying $\psi$ to all vertices simultaneously, we obtain a mapping $\Psi: \mathcal{P}^{k+3} \rightarrow \mathcal{P}_{\text {reduced }}^{k+3}$. Note that the isomorphism $\psi$ depends on the polygon $P \in \mathcal{P}^{k+3}$. The mapping $\Psi$ will be proved to be a stable composition. Steps 1 and 2 together imply the lemma. This is due to the fact that the projective isomorphism $\psi$ transfers the points $\widetilde{q}_{i}$ defined by $P \in \mathcal{P}^{k+3}$ to the corresponding points defined by $\Psi(P)$. Hence the composition (of both steps) $\xi \circ \Psi: \mathcal{P}^{k+3} \rightarrow \mathcal{P}_{\text {reduced }}^{k+3} \rightarrow \mathbb{R}_{\text {ordered }}^{k}$ in fact coincides with the direct application of $\xi$.

Step 1. Note that the mapping $\Psi$ is well defined and onto. Let $L_{\infty} \subset \mathbb{P}^{2}$ denote the line at infinity. We parametrize the space $\mathcal{P}^{k+3}$ by means of the equivalence class (in $\mathcal{P}_{\text {reduced }}^{k+3}$ ) and the corresponding isomorphism $\psi$. The polygons in $\mathcal{P}^{k+3}$ are then given by $\psi^{-1}(P)$, where $(P, \psi)$ is an admissible pair formed by an equivalence class $P \in \mathcal{P}_{\text {reduced }}^{k+3}$ and a projective isomorphism $\psi$. Such a pair is called admissible if the following conditions are satisfied:

- $(0,0),(1,0)$, and $(0,1)$ are fixed points of $\psi$.

- $\psi\left(L_{\infty}\right) \cap P=\varnothing$.

A moment's reflection shows that the space $\mathcal{P}^{k+3}$ is rationally equivalent to the set of the admissible pairs $(P, \psi)$. The space of the projective isomorphisms with the desired fixed points can be parametrized by means of the points $c$ and $d$, which arise by intersection of $\psi\left(L_{\infty}\right)$ with $\operatorname{proj}\left(p_{\infty}, p_{0}\right)$ and $\operatorname{proj}\left(p_{\infty}, p_{k+1}\right)$, respectively. The admissible isomorphisms among them are then characterized by the property $[(c, 0),(0, d)] \cap P=\emptyset$; see Fig. 6. Note that $c, d \notin[0,1]$. In order to avoid values of $c$ and $d$ at infinity we use another 


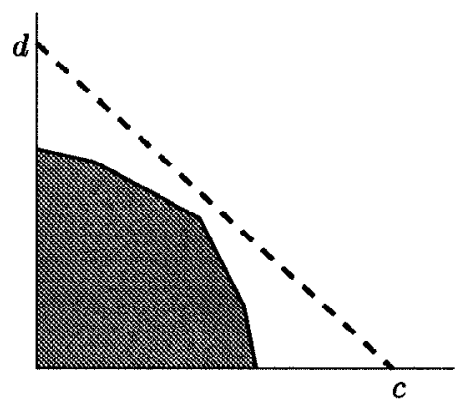

Fig. 6. An admissible image $\psi\left(L_{\infty}\right)$.

rational equivalence, induced by a special projective transformation $\bar{\psi}$. The isomorphism $\bar{\psi}$ is defined by sending $(0,0) \mapsto(0,0),\left(\frac{1}{2}, 0\right) \mapsto\left(-\frac{1}{2}, 0\right),\left(0, \frac{1}{2}\right) \mapsto\left(0,-\frac{1}{2}\right)$, and both points $p_{0}$ and $p_{k+1}$ to the line at infinity. Now the space of admissible $\psi$ is reparametrized by those pairs $(\alpha, \beta)$ of positive reals such that $[(\alpha, 0),(0, \beta)] \cap \bar{\psi}(P)=\emptyset$; see Fig. 7 . (Note that $\bar{\psi} \circ \psi\left(L_{\infty}\right)=\operatorname{proj}((\alpha, 0),(0, \beta))$. The image $\bar{\psi}(P)$ is shaded.) For given $P$ and $\alpha$ the space of admissible $\beta$ is therefore determined by the inequality system $(\beta, \alpha) * p_{i}>\alpha \beta, i=1, \ldots, k$, where “*” stands for the scalar product.

Step 2 . The space $\mathcal{P}_{\text {reduced }}^{k+3}$ is parametrized by the variables $q_{i}$ and $r_{i}$, for $i=0, \ldots, k$. The values of $q_{i}$ and $r_{k-i}$ are defined by the intersection points of aff $\left(p_{i}, p_{i+1}\right)$ with the coordinate lines. Note that the vector $\left(q_{1}, \ldots, q_{k}\right)$ coincides with the image of the decoding mapping $\xi(P)$. Therefore it suffices to show that the mapping $\left(\left(q_{0}, \ldots, q_{k}\right)\right.$, $\left.\left(r_{0}, r_{i}, r_{i+1}, \ldots, r_{k}\right)\right) \mapsto\left(\left(q_{0}, \ldots, q_{k}\right),\left(r_{0}, r_{i+1}, \ldots, r_{k}\right)\right)$ of forgetting the entry $r_{i}$ constitutes a stable projection for any index $i=1,2, \ldots, k-1$. See Fig. 8 for an interpretation of the latter projection mapping as a mapping $\mathcal{P}_{\text {reduced }}^{k+3-(i-1)} \rightarrow \mathcal{P}_{\text {reduced }}^{k+3-i}$. The following manipulation of the polygon is used. Starting from $\widetilde{p}_{k}:=p_{k}$ and $i=1$ we consider the polygon $\left(p_{\infty}, p_{0}, p_{1}, \ldots, p_{k-i}, \widetilde{p}_{k-i+1}, p_{k+1}\right)$. Forgetting the entry $r_{i}$ finds its equivalent in removing the edge $\left(p_{k-i}, \widetilde{p}_{k-i+1}\right)$. Then $\widetilde{p}_{k-i}$ is defined to be the intersection point of the prolonged neighboring edges. We show that the set of possible entries $r_{i}$ can be recovered from the result of the projection mapping by means of two polynomial constraints. First, we have $r_{i}>r_{0}=1$. Second, the point $\widetilde{p}_{k-i}$ must lie "above" the line

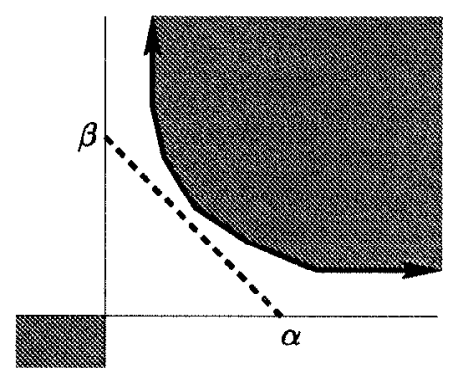

Fig. 7. The image $\bar{\psi} \circ \psi\left(L_{\infty}\right)$. 


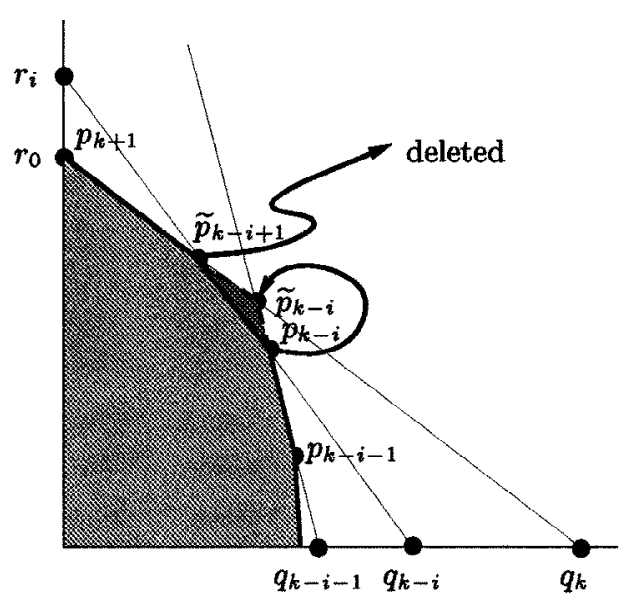

Fig. 8. Forgetting $r_{i}$.

aff $\left(\left(q_{k-i}, 0\right),\left(0, r_{i}\right)\right)$. This is equivalent to $\left(r_{i}, q_{k-i}\right) * \widetilde{p}_{k-i}>r_{i} q_{k-i}$. By Cramer's rule the coordinates of $\widetilde{p}_{k-i}$ are rational functions of the vectors $q$ and $r$. The denominator function provided by Cramer's rule does not vanish for geometrical reasons: in fact the point $\widetilde{p}_{k-i}$ does not belong to the line at infinity. Multiplication of the above inequality with the square of the denominator provides the second polynomial constraint. Note that the application of Cramer's rule can be substituted by appropriate rational equivalences, as performed in Step 1. However, here such a technique would considerably complicate the exposition.

\subsection{The Starting Polytope}

In the previous section we have seen that a $(k+3)$-gon encodes an ordered $k$-tuple in view of the mapping $\xi$. This section is devoted to duplicating the encoding polygon in order to make it accessible to further manipulations. Generally speaking, we use a "bundled" product of an encoding $(k+3)$-gon $P \in \mathcal{P}^{k+3}$ with an "admissible" $l$-gon. Then $l-2$ copies of the encoding polygon are obtained. Suppose that $l \geq 4$. In the present framework it is more convenient to consider realizations of a polygon not as subsets of $\mathbb{R}^{2}$, but as subsets of an affine 2-subspace of $\mathbb{R}^{4}$. For the realizations of the encoding polygon we use the affine space given by the base $p_{\infty}:=(0,0,0,0)$, $p_{0}:=(1,0,0,0)$, and $p_{k+1}:=(0,1,0,0)$. Realizations of the $l$-gon $R=\left(r_{1}, \ldots, r_{l}\right)$ are considered as subsets of the affine space $A$ given by the base $r_{1}:=(0,0,1,0)$, $r_{2}:=(0,0,0,1)$, and $r_{3}:=(0,1,0,0)$. For any pair $(P, R)$ the following construction can be performed. First, we fix a projective isomorphism $\varphi$ on $\mathbb{R}^{4}$ such that the four points $(0,0,0,0),(1,0,0,0),(0,0,1,0)$, and $(0,0,0,1)$ are sent to the 3 -space at infinity and the common point $p_{k+1}=r_{3}=(0,1,0,0)$ of $P$ and $R$ to the origin. For an easier formulation of the construction we suppose that the images of $(0,0,0,0)$ and $(1,0,0,0)$ are in the projective closure of $\mathbb{R}^{2} \times(0,0)$ and the images of $(0,0,1,0)$ and $(0,0,0,1)$ 
are in the projective closure of $(0,0) \times \mathbb{R}^{2}$. (In fact, the construction is independent of the special choice of $\varphi$.) Second, let $L_{\infty}$ denote the original space at infinity. Note that $\varphi\left(L_{\infty}\right)$ intersects neither $\varphi(P)$ nor $\varphi(R)$. Third, we consider the images $\varphi(P) \subset \mathbb{R}^{2} \times(0,0)$ and $\varphi(R) \subset(0,0) \times \mathbb{R}^{2}$. Both images are unbounded 2-polyhedra each with two vertices fewer that the original. What we want is to treat the sum of the latter 2-polyhedra, but only if the $l$-gon $R$ satisfies a special condition, namely that all vertices $r_{3}, \ldots, r_{l}$ are admissible points. A point $r \in A$ is called admissible if $r_{1}, r_{2}, r_{3}, r$ are the vertices of a quadrilateral set appearing in the given order and the sum $\varphi(P)+\varphi(r)$ does not intersect the image $\varphi\left(L_{\infty}\right)$ of the space at infinity. Using similar arguments to those in the proof of Lemma 9, we see that the set of admissible points is the relative interior of a polygon which can be described by linear inequalities whose coefficients depend polynomially on $P$ only. Assuming the vertices $r_{3}, \ldots, r_{l}$ to be admissible points, the bundled product of $P$ and $R$ is defined to be the inverse image (under $\varphi$ ) of the sum $\varphi(P)+\varphi(R)$. The canonical projection from the space of bundled products (of some encoding $(k+3)$ gon with an $l$-gon) to the space $\mathcal{P}^{k+3}$ of encoding $(k+3)$-gons constitutes a stable composition.

Now let $l:=3+r+m$; recall that $r$ denotes the number of conditions and $m$ the number of sign-functions used in Lemma 8 . The space of bundled products coincides with the realization space of a 4-polytope called the starting polytope (provided that $l$ is the right number, namely $l=3+r+m$ ). In fact, the face lattice of every bundled product is the same. This is directly implied by its definition as the inverse image of a sum. The face lattice is obtained from the face lattice of the Cartesian product by a contraction of $\left\{p_{\infty}\right\} \times R,\left\{p_{0}\right\} \times R, P \times\left\{r_{1}\right\}$ and $P \times\left\{r_{2}\right\}$.

On the other hand, any polytope with the described face lattice is a bundled product, i.e., it can be obtained by the above construction. This property appears to be the decisive property; it says that an entire realization space (of 4-polytopes) is covered by the construction. In fact, if we start with an arbitrary polytope combinatorially equivalent to a bundled product, then the transformation $\varphi$ can be recovered. (It is given by the position of the (well-defined) vertices corresponding to the images of $(0,0,0,0),(1,0,0,0),(0,0,1,0)$, and $(0,0,0,1)$.) The images of the 2-faces bundled at $[(0,0,0,0),(1,0,0,0)]$ (the encoding polygons) are parallel. The same is true for the images of the 2 -faces bundled at $[(0,0,1,0),(0,0,0,1)]$. Now it is a property of the face lattice - of the polyhedron which is obtained as the image of the given 4-polytope-that it is a sum in the sense of the construction. This implies that the considered polytope is a bundled product. Here (and in the following), the notation $[A, B]$ stands for the convex hull of two given convex sets $A$ and $B$.

How should we refer to vertices and faces of the starting polytope in a convenient way? We can make use of the (bundled) product structure. A vertex corresponding to the pair $\left(p_{i}, r_{j}\right)$ (after the application of $\varphi$ ) is denoted by $p_{i}^{j}$. We can regard it as a copy of the vertex $p_{i}$ in a copy of the encoding polygon, namely in the copy with the number $j$. The points $p_{\infty}^{j}$ and $p_{0}^{j}$ coincide for all copies (this is the sense of bundling), therefore we delete the upper index $j$ for these vertices. The encoding polygon with upper index $j$ is abbreviated by $P^{j}$. Figure 9 shows what a Schlegel diagram of a starting polytope in principle looks like. (For details on Schlegel diagrams we refer to [14].) 


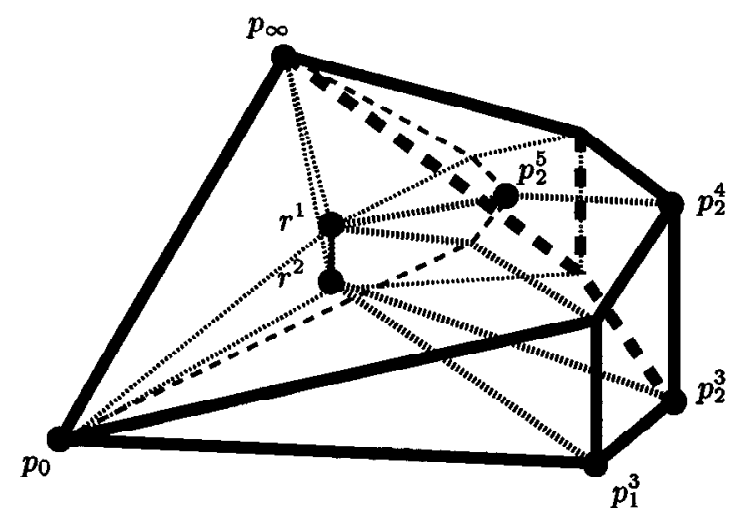

Fig. 9. The starting polytope.

\subsection{Outline of the Polytopal Part of the Construction}

We consider a specific two-dimensional projection of the starting polytope to explain the ideas of the following constructions. The projection we use is $\varphi^{-1} \circ \Pi \circ \varphi$, where $\Pi$ stands for the orthogonal projection $\Pi: \mathbb{R}^{4} \rightarrow\left\{(0,0) \times \mathbb{R}^{2}\right\}$, and $\varphi$ is the projective isomorphism on $\mathbb{P}^{4}$ used in the definition of the bundled product in Section 3.4. The projection of an encoding polygon $P^{j}$ is just one point, namely the vertex $r_{j}$ of the $l$-gon $R$. The projection of the starting polytope is the entire $l$-gon. Using this projection the encoding polygons are ordered in a geometrical (not only abstract) setting. Of special interest are the edges $\left[r_{j}, r_{j+1}\right], j=3, \ldots, 2+r+m$, of the projection, each corresponding to a facet $\left[P^{j}, P^{j+1}\right]$ of the starting polytope. Note that there are as many of these edges as there are conditions and sign-functions. Let a fixed one-to-one correspondence be given. Between the encoding $(k+3)$-gons $P^{j}$ and $P^{j+1}$ we attach hexagons; where the term "between" is understood in connection with the geometrical order given by the projection. The hexagons are not supposed to encode the entire $k$-tuple of ordered points on the real line we have in mind. Their job is just to encode the pair of ordered triples that are compared by the condition or sign-function we are treating. (In the case of a sign-function the interpretation is slightly different.) The attaching can be made "invisible" for the rest of the polytope by means of the connected sum operation as explained by Richter-Gebert [9]. This is important in order to determine the face lattice of the extended polytope. The convex hull of the two attached hexagons forms a facet of the extended polytope. The major advantage of the use of hexagons consists of the fact that the face lattice of this new facet will be the same in all cases. The last step of the construction is the attachment of a von Staudt polytope to the latter facet. The von Staudt polytope is the part of the construction converting a condition or sign-function into a property of the face lattice of the polytope.

To describe the following steps of the proof we need two polytopal tools, called "attaching an encoding hexagon" and "attaching a von Staudt polytope." Besides this we have to keep control of the face lattice of 3-polytopes (facets) which arise as the convex 


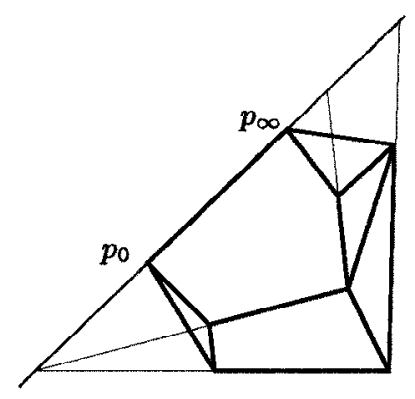

Fig. 10. Two bundled polygons.

hull of polygons encoding ordered $k^{\prime}$-tuples of possibly different size. The latter is what we begin with.

\subsection{Bundled Polygons and Order Types}

Lemma 10. Let $P^{1}=\left(p_{\infty}, p_{0}, p_{1}^{1}, \ldots, p_{k^{1}+1}^{1}\right)$ and $P^{2}=\left(p_{\infty}, p_{0}, p_{1}^{2}, \ldots, p_{k^{2}+1}^{2}\right)$ be polygons in $\mathbb{R}^{4}$ with distinct affine hulls, which are bundled at the common edge $\left[p_{\infty}, p_{0}\right]$; see Fig. 10. Then the convex hull $P:=\left[P^{1}, P^{2}\right]$ of both polygons has dimension 3. The encoded sets of ordered points on the real line are denoted by $q^{1}:=\xi\left(P^{1}\right)$ and $q^{2}:=\xi\left(P^{2}\right)$, respectively. The mapping $\Theta:\left\{1, \ldots, k^{1}\right\} \times\left\{1, \ldots, k^{2}\right\} \rightarrow\{-, 0,+\}$ defined by

$$
\Theta(i, j):=\left\{\begin{array}{lll}
- & \text { if } & q_{j}^{1}<q_{i}^{2}, \\
0 & \text { if } q_{i}^{1}=q_{j}^{2}, \\
+ & \text { if } q_{i}^{1}>q_{j}^{2}
\end{array}\right.
$$

is called the order type of $\left(q^{1}, q^{2}\right)$. (Here, the image of $\xi$ in $\mathbb{R}$ is used for convenience.) Then the face lattice $\mathcal{L}(P)$ is a well-defined injective function of the order type $\Theta\left(q^{1}, q^{2}\right)$.

Proof. We consider the linear objective function $f_{c}(x):=c * x$, where $c \not \perp P^{1}, c \not \perp P^{2}$. A vertex $p_{j}^{1}, j=1, \ldots, k^{1}$, is optimal for some $f_{c}$ if and only if $q_{j}^{1}(c)$ belongs to the interval $\left[q_{j-1}^{1}, q_{j}^{1}\right]$. Here, put $\widetilde{q}_{j}^{1}(c):=\operatorname{proj}\left(p_{\infty}, p_{0}\right) \cap\left\{x \mid f_{c}(x)=f_{c}\left(p_{j}^{1}\right)\right\}$ (see Fig. 11), and let $q_{j}^{1}(c)$ be the image of $\widetilde{q}_{j}^{1}(c)$ under the projective transformation that defines $q$ as an image of $\tilde{q}$; compare Section 3.3. On the other hand, the vertices $p_{j}^{1}$ and $p_{i}^{2}$ are incident with a common facet if and only if both vertices are maximal points for the same $f_{c}$. The latter is equivalent to the intersection $\left[q_{j-1}^{1}, q_{j}^{1}\right] \cap\left[q_{i-1}^{2}, q_{i}^{2}\right]$ being nonempty. We conclude that the order type determines the face lattice of $\left[P^{1}, P^{2}\right]$, and vice versa.

Corollary 11. Let $P^{1}, P^{2}$, and $P^{3}$ be polygons in $\mathbb{R}^{4}$ bundled at the common edge $\left[p_{\infty}, p_{0}\right]$. Assume that the convex hull $P=\left[P^{1}, P^{2}, P^{3}\right]$ is of dimension 4 . Then the 


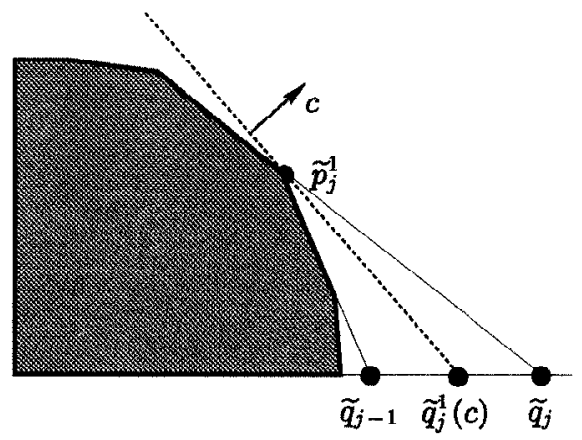

Fig. 11. Definition of $\widetilde{q}_{j}^{1}(c)$.

combinatorial type $\mathcal{L}(P)$ is a well-defined injective function of the corresponding triple of order types.

\subsection{Invisible Regions}

Definition 12. Let $P$ and $\widetilde{P}$ denote the vertex vectors of a 4-polytope in $\mathbb{R}^{4}$ and one of its facets. Then the invisible region $\mathcal{I}(P, \widetilde{P})$ of $P$ generated by $\widetilde{P}$ is defined to be the set of those points in $\mathbb{R}^{4}$ which lie beyond $\widetilde{P}$ and beneath all the other facets of $P$.

In Fig. 12 we have two typical examples of an invisible region; note that $\mathcal{I}$ is always the interior of a polyhedral set. For a precise definition of the beneath-beyond terminology we refer to [3].

Lemma 13. Consider a subset $M \subset \mathbb{R}^{4 k}$ such that any $P=\left(p_{1}, \ldots, p_{k}\right) \in M$ is the vertex vector of a 4-polytope and the projection $\widetilde{P}$ is the vertex vector of one of its facets. (The combinatorial types of $P$ and $\widetilde{P}$ are not required to be constant on $M$.) Then the space

$$
\tilde{M}:=\left\{\left(P, p_{k+1}\right) \in M \times \mathbb{R}^{4} \mid p_{k+1} \in \mathcal{I}(P, \widetilde{P})\right\}
$$
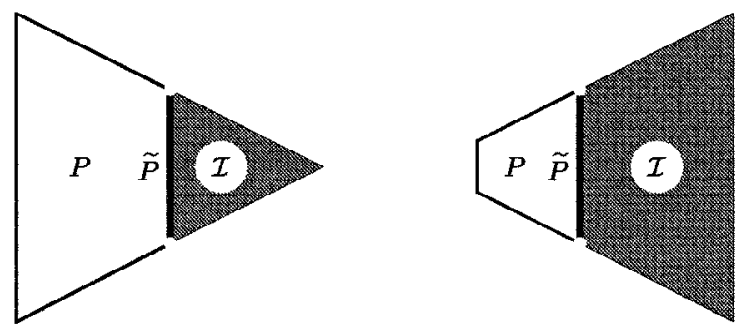

Fig. 12. Typical invisible regions. 
is stably equivalent to $M$, and the projection mapping (of forgetting the new point) $\widetilde{M} \rightarrow M$ given by $\left(P, p_{k+1}\right) \mapsto P$ is a stable projection.

Idea of Proof. The proof uses the fact that the admissible positions of $p_{k+1}$ are described by strict linear inequalities whose coefficients are rational functions of $P$. The definition of the latter inequalities (applying Cramer's rule), and the arguments leading to polynomial coefficients, follow the same lines as in the proof of Lemma 9 (Step 2).

Now instead of a singleton only, we want to place an $l$-tuple of points within the invisible region. To this end we use the additional assumption that the "construction" of the $l$-tuple is projectively unique with respect to the facet $\widetilde{P}$. There are important examples for such a situation. Think of the Lawrence extension (see [14]). Also the von Staudt polytope to be defined below turns out to be projectively unique with respect to its basic facet.

Corollary 14. Let the assumptions of Lemma 13 hold. Let $\Pi_{M}: \mathbb{R}^{4 k} \rightarrow \mathbb{R}^{4 k^{\prime}}$ denote the canonical projection $P \mapsto \widetilde{P}$. (The vertex vector of $P$ is supposed to start with the vertices of $\widetilde{P}$.) Consider a polynomial mapping $\mathbb{R}^{4 k^{\prime}} \rightarrow \mathbb{R}^{4 k^{\prime}+4 l}$ (with integer coefficients) associating to any facet $\widetilde{P}$ a polytope $\bar{P}(\widetilde{P})$ containing $\widetilde{P}$ as a facet. (The image stands for the equivalence class of possible constructions.) Define the space $\widetilde{M}$ of invisible attachings of $\bar{P}$ to $P$ as follows:

$$
\left\{\begin{array}{l|l}
\tilde{M}:=\left\{\left(p_{1}, \ldots, p_{k+l}\right) \in \mathbb{R}^{4 k+4 l}\right. & \begin{array}{l}
\left(p_{1}, \ldots, p_{k}\right) \in M, \\
\left(p_{1}, \ldots, p_{k^{\prime}}, p_{k+1}, \ldots, p_{k+l}\right) \approx \bar{P}(\widetilde{P}), \\
p_{k+1}, \ldots, p_{k+l} \in \mathcal{I}(P, \tilde{P})
\end{array}
\end{array}\right\},
$$

where the symbol $\approx$ abbreviates projective equivalence. Then the space $\tilde{M}$ is stably equivalent to $M$ and the projection mapping (of forgetting the new points) $\widetilde{M} \rightarrow M$, $\left(p_{1}, \ldots, p_{k+l}\right) \mapsto P$ is a stable projection.

Idea of Proof. The proof uses two vertices of $\bar{P}(\widetilde{P})$ that form a projective basis of $\mathbb{R}^{4}$ together with an affine basis of $\widetilde{P}$. Then all the vertices of the attached polytope are rational functions of $\widetilde{P}$ and the position of the latter pair of vertices. The rest of the proof follows the same lines as in Lemma 13.

In view of the program of the proof and Lemma 13 one can already imagine what is meant by "attaching an encoding hexagon." Details are given in Section 3.9.

\subsection{The von Staudt Polytope}

Step 1: The Basic Facet. Consider a pair of encoding hexagons $P^{1}$ and $P^{2}$ in $\mathbb{R}^{4}$ bundled at the common edge $\left[p_{\infty}, p_{0}\right]$. The 3 -polytope $\left[P^{1}, P^{2}\right]$ is called a basic facet (of the 


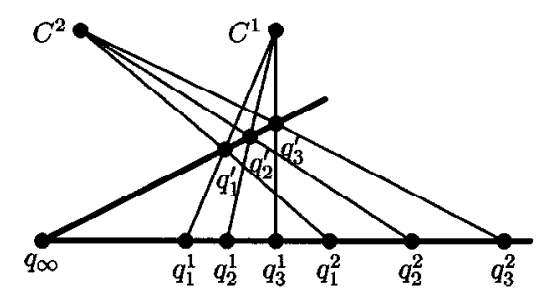

Fig. 13. Projective equivalence using two perspectivities.

construction) if the following conditions hold:

(i) $\left(q_{1}^{1}, q_{2}^{1}, q_{3}^{1}, q_{1}^{2}, q_{2}^{2}, q_{3}^{2}\right)$ forms an ordered 6-tuple, where $q_{j}^{i}:=\xi_{j}\left(P^{i}\right)$.

(ii) The pair of ordered triples defined by the hexagons satisfies the condition $C$ : $\left(q_{1}^{1}, q_{2}^{1}, q_{3}^{1}\right) \approx\left(q_{1}^{2}, q_{2}^{2}, q_{3}^{2}\right)$.

Step 2: The Perspectivities: Definition on the Bundling Line. The condition C in (ii) (stated on the real line image of $\xi$ ) reads as follows if we treat the geometrical image of $\xi$ (the points of intersection of the affine hulls of the edges with the bundling line): there exists a projective isomorphism on the bundling line sending $q_{\infty} \mapsto q_{\infty}, q_{1}^{1} \mapsto q_{1}^{2}, q_{2}^{1} \mapsto q_{2}^{2}$, and $q_{3}^{1} \mapsto q_{3}^{2}$. Similar to von Staudt constructions, the latter projective isomorphism can be obtained by a composition of two perspectivities (in $\overline{\mathbb{R}^{4}}$ ), namely $q_{\infty} \mapsto q_{\infty} \mapsto q_{\infty}$, $q_{1}^{1} \mapsto q_{1}^{\prime} \mapsto q_{1}^{2}, q_{2}^{1} \mapsto q_{2}^{\prime} \mapsto q_{2}^{2}, q_{3}^{1} \mapsto q_{3}^{\prime} \mapsto q_{3}^{2}$; see Fig. 13 .

Both perspectivities must preserve the order of the points in a certain sense. The pair of perspectivities is called admissible if $q_{1}^{\prime} \in \mathbb{R}^{4}$ (not at infinity) and $q_{2}^{\prime} \notin\left(q_{\infty}, q_{1}^{\prime}\right)$. The condition $q_{2}^{\prime} \notin\left(q_{\infty}, q_{1}^{\prime}\right)$ implies that the projective lines defining $C^{1}$ as their intersection point (namely those connecting $q_{1}^{1}$ with $q_{1}^{\prime}$ and $q_{2}^{1}$ with $q_{2}^{\prime}$ ) do not intersect on the open line segment $\left(q_{1}^{1}, q_{1}^{\prime}\right)$; this is due to the fact that $\left(q_{1}^{1}, q_{2}^{1}\right)$ is an ordered pair. An analogous assertion holds true for $C^{2}$. This property is referred to as the orientation property. Note that the construction is projectively unique. (This follows from the fact that that $q_{1}^{1}, q_{2}^{1}$, $q_{1}^{\prime}, q_{2}^{\prime}$ form the projective basis of a 2-space.)

Step 3: The Perspectivities: Application to the Encoding Hexagons. This is the crucial step of the construction. The aim is to fix the perspectivities defined in Step 2 by polytopal means. The idea is to apply them to the encoding hexagons, in fact they are applied to the "pentagons" $\widetilde{P}^{i}:=\left(p_{\infty}, q_{1}^{i}, p_{2}^{i}, p_{3}^{i}, p_{4}^{i}\right), i=1,2$ (at least after suitable projective transformations they are convex sets). What we want is to use the first perspectivity for defining a projective equivalent of $\widetilde{P}^{1}$ somewhere in $\mathbb{R}^{4}$, and to copy the latter equivalent to $\widetilde{P}^{2}$ by means of the second perspectivity. The presence of the perspectivities can then be fixed by Lawrence extensions. (The point $q_{1}^{\prime}$ must be chosen outside the affine hull of the basic facet.)

However, the construction cannot be done that (two step) way, not even under the (possible) assumption that the pentagons $\widetilde{P}^{1}$ and $\widetilde{P}^{2}$ are projectively equivalent. The impossibility is implied by the fact that the projective hull of $P^{1}, C^{1}$, and $C^{2}$ is only a 3-space, hence $\widetilde{P}^{2}$ would not be covered.

Therefore we use three steps, i.e., a copy $P^{3}$ of $\widetilde{P}^{1}$ and a different copy $P^{4}$ of $\widetilde{P}^{2}$ 


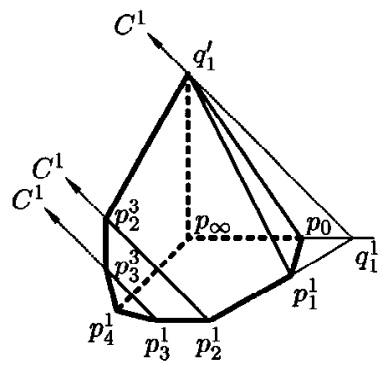

Fig. 14. A perspectivity between polygons.

being induced by the perspectivities with the centers $C^{1}$ and $C^{2}$, respectively. Details (on the choice of their affine hulls) follow below. The copies $P^{3}$ and $P^{4}$ can even be supposed to be bundled at the line $\operatorname{aff}\left(p_{\infty}, q_{1}^{\prime}\right)$. Then, in view of Lemma 10, we do not need a perspectivity between them to compare the encoded triples, i.e., no additional assumption is required concerning the shape of $\widetilde{P}^{1}$ and $\widetilde{P}^{2}$.

The pentagon $P^{3}$ is defined as follows ( $P^{4}$ analogously). First, we define its affine hull by setting $A^{3}:=\operatorname{aff}\left(p_{\infty}, q_{1}^{\prime}, p_{4}^{1}\right)$. Note that $C^{1}$ belongs to the projective hull of $P^{1}$ and $q_{1}^{\prime}$. Let $p_{2}^{3}, p_{3}^{3}$ then be the images of $p_{2}^{1}, p_{3}^{1}$ under the perspectivity between aff $P^{1}$ and $A^{3}$ induced by $C^{1}$; see Fig. 14 . Now the vertex vector of $P^{3}$ is defined by setting $P^{3}:=\left(p_{\infty}, q_{1}^{\prime}, p_{2}^{3}, p_{3}^{3}, p_{4}^{1}\right)$.

If both $P^{3}$ and $P^{4}$ are the vertex vector of pentagons (convexity), then the resulting 4-polytope $P:=\left[P^{1}, P^{2}, P^{3}, P^{4}\right]$ is called a von Staudt polytope.

Is the von Staudt polytope well defined? To see this, recall that the entire construction is projectively unique for given basic facet $\left[P^{1}, P^{2}\right]$. Thus it suffices to show the existence of a pair of convex images $P^{3}$ and $P^{4}$. In fact, both centers of perspectivity $C^{1}$ and $C^{2}$ are arbitrarily close to the line $\operatorname{aff}\left(p_{\infty}, q_{1}^{\prime}\right)$ provided that $q_{2}^{\prime}$ is sufficiently close to $q_{1}^{\prime}$. The orientation property forces the convexity of $P^{3}, P^{4}$, for such choices of $C^{1}, C^{2}$, indeed.

Step 4: The Perspectivities: Stabilization by Lawrence Extensions. In order to make the construction recoverable from the face lattice (of its result) the perspectivities are stabilized by Lawrence extensions. In case of $C^{1}$ the Lawrence extension is defined as follows; see also [14]. The construction starts with $P^{1}, P^{3}$, and the center of perspectivity $C^{1}$. Then two points $p_{\{1,3\}}, p_{\{1,3\}}^{\prime} \in \mathbb{R}^{4} \backslash \operatorname{aff}\left(P^{1}, P^{3}\right)$ are added such that

- $C^{1} \in \operatorname{proj}\left(p_{\{1,3\}}, p_{\{1,3\}}^{\prime}\right)$,

- $C^{1} \notin\left[p_{\{1,3\}}, p_{\{1,3\}}^{\prime}\right]$,

- the vertices of the quadrilateral set $\left(p_{\{1,3\}}, p_{\{1,3\}}^{\prime}, q_{1}^{\prime}, q_{1}^{1}\right)$ appear in the described order.

The latter construction is known as a Lawrence extension (see, e.g., [14]). It is not hard to see that the face lattice of $\left[P^{1}, P^{3}, p_{\{1,3\}}, p_{\{1,3\}}^{\prime}\right]$ is the same for all possible constructions, and that the point $C^{1}$ can be recovered for all realizations of the latter 
face lattice as the intersection point of $\operatorname{proj}\left(p_{3}^{1}, p_{3}^{3}\right)$ and $\operatorname{proj}\left(p_{2}^{1}, p_{2}^{3}\right)$, see [14] and [9]. We prove that $C^{1} \in \operatorname{proj}\left(q_{1}^{1}, q_{1}^{\prime}\right)$ is also implied by the face lattice. In fact, $C^{1}$ is the intersection point of $\operatorname{proj}\left(p_{\{1,3\}}, p_{\{1,3\}}^{\prime}\right)$ with the 3 -space $\operatorname{proj}\left(P^{1}, P^{3}\right)$ defined to be the projective hull of $P^{1}$ and $P^{3}$. The face lattice of the Lawrence extension exhibits a facet with the vertices $p_{\infty}, p_{0}, q_{1}^{\prime}, p_{\{1,3\}}, p_{\{1,3\}}^{\prime}$. Therefore $\operatorname{proj}\left(p_{\{1,3\}}, p_{\{1,3\}}^{\prime}\right)$ intersects the 2-space $\operatorname{proj}\left(p_{\infty}, p_{0}, q_{1}^{\prime}\right) \subset \operatorname{proj}\left(P^{1}, P^{3}\right)$. The intersection point must be $C^{1}$. The point $C^{1}$ thus belongs to the 2 -space $\operatorname{proj}\left(p_{\infty}, p_{0}, q_{1}^{\prime}\right)$. It also belongs to the 2 -space $\operatorname{proj}\left(p_{1}^{1}, p_{2}^{1}, p_{2}^{3}, q_{1}^{\prime}\right)$. The intersection of the latter 2 -spaces is $\operatorname{proj}\left(q_{1}^{1}, q_{1}^{\prime}\right)$, which implies the assertion.

A von Staudt polytope $P$ endowed with Lawrence extensions of the perspectivities (of $C^{1}$ and $C^{2}$ ) is called an extended von Staudt polytope if the new points are in the corresponding invisible regions, i.e., $p_{\{1,3\}}, p_{\{1,3\}}^{\prime} \in \mathcal{I}\left(P,\left[P^{1}, P^{3}\right]\right)$ and $p_{\{2,4\}}, p_{\{2,4\}}^{\prime} \in$ $\mathcal{I}\left(\left[P,\left\{p_{\{1,3\}}, p_{\{1,3\}}\right\}\right],\left[P^{2}, P^{4}\right]\right)$.

Lemma 15. From any basic facet (in the sense of Step 1) there can be constructed an extended von Staudt polytope, where the construction of the von Staudt polytope is projectively unique. The face lattices of all (extended) von Staudt polytopes are the same. Conversely, any realization (of the face lattice) of an extended von Staudt polytope is the result of the latter construction process starting with its basic facet. (The construction can be inverted.) In particular, any basic facet of a (realization of a) von Staudt polytope is a basic facet of the construction in the sense of Step 1.

Idea of Proof. A moment's reflection shows that the space of basic facets (in the sense of Step 1) has trivial topology. In order to see the coincidence of the face lattices it therefore suffices to prove the matroids (defined by affine dependence) of the vertices to coincide for all the constructible von Staudt polytopes. This is not a hard task for the given construction. However, the computability of the face lattice-as stated in the Main Theorem-requires knowledge of the lattice. This could be established by means of an example with rational coefficients. In fact, the Universal Partition Theorem does not need more care of the von Staudt polytope's face lattice. For the reader to use the computation of a (numerical) example in connection with a structural theorem might be unsatisfactory. The reader might also want to see the result of a geometrical construction as a real geometrical object (with all its combinatorial properties) actually presented. To meet these wishes a structural analysis of the face lattice of the von Staudt polytope is presented in Section 4.

Why is the construction invertible? First, the (combinatorial equivalent of) the basic facet is present in all realizations of the (extended) von Staudt polytope. In view of Lemma 10 this implies condition (i) of Step 1. Second, the perspectivities $C^{1}$ and $C^{2}$ are present, as already seen in Step 5. Third, it remains to prove condition (ii) of Step 1 holds. The application of the perspectivities do not require the latter condition. Assuming condition (ii) is not satisfied, the images of $q_{3}^{1}$ and $q_{3}^{2}$ on $\operatorname{proj}\left(p_{\infty}, q_{1}^{\prime}\right)$ (under the perspectivities $C^{1}$ and $C^{2}$, respectively) could not coincide. This in view of Lemma $10 \mathrm{implied}$ the face lattice of the facet $\left[P^{3}, P^{4}\right]$ to be distinct from the corresponding facet's lattice of a constructible von Staudt polytope. Hence such a polytope cannot be a realization of the (combinatorial) von Staudt polytope. 


\subsection{Putting Everything Together}

The basis of the construction is the starting polytope $P$. As in Section 3.4, we fix a one-to-one correspondence between the set of conditions and sign-functions on the one hand, and the set of facets $P^{j}, P^{j+1}, j=3, \ldots, 2+r+m$, of the starting polytope on the other. By induction over $j$ the polytope $P$ is modified in order to encode the condition or sign-function corresponding to $j$ as a property of the face lattice. Suppose that the condition or sign-function corresponding to the index $j$ compares the ordered triples $\left(q_{1}^{1}, q_{2}^{1}, q_{3}^{1}\right)$ and $\left(q_{1}^{2}, q_{2}^{2}, q_{3}^{2}\right)$.

First, a hexagon $P^{1}=\left(q_{\infty}, q_{0}, p_{1}^{1}, p_{2}^{1}, p_{3}^{1}, p_{4}^{1}\right)$ encoding the triple $\left(q_{1}^{1}, q_{2}^{1}, q_{3}^{1}\right)$ is chosen in such a way that the new points $p_{1}^{1}, p_{2}^{1}, p_{3}^{1}, p_{4}^{1}$ belong to the invisible region $\mathcal{I}\left(P,\left[P^{j}, P^{j+1}\right]\right)$ defined by the facet $\left[P^{j}, P^{j+1}\right]$.

The operation is called attaching an encoding polygon. The inverse operation of forgetting the new points is a mapping from the space of possible constructions to the space of polytopes the attaching has started with. It constitutes a stable composition. In fact, we can choose any projective equivalence class for the hexagon to be attached. This choice corresponds to a stable composition as seen in Lemma 9. Given the equivalence class, it remains to determine the space of embeddings such that all the new points belong to the invisible region. This corresponds to a stable projection in view of Corollary 14.

The second step depends on whether a condition or a sign-function is involved. If we deal with a condition, then a hexagon $P^{2}$ encoding the triple $\left(q_{1}^{2}, q_{2}^{2}, q_{3}^{2}\right)$ is attached to $\left[P^{1}, P^{j+1}\right]$. If a sign-function is treated, then the same hexagon is attached, but it is labeled by $P_{0}^{2}$. After this, a hexagon $P^{2}$ is attached to $\left[P^{1}, P_{0}^{2}\right]$, which encodes the triple $\left(q_{1}^{2}, q_{2}^{2}, \varphi\left(q_{3}^{1}\right)\right)$, where $\varphi$ denotes the projective isomorphism on the line sending $q_{1}^{1} \mapsto q_{1}^{2}, q_{2}^{1} \mapsto q_{2}^{2}$, and $q_{\infty} \mapsto q_{\infty}$

In any case after two or three attachings we obtain a modified polytope exhibiting a facet $\left[P^{1}, P^{2}\right]$, which satisfies all the conditions for the basic facet of a von Staudt polytope. The next step is to attach a von Staudt polytope such that the new points belong to the invisible region corresponding to $\left[P^{1}, P^{2}\right]$. Again, the inverse operation of forgetting the new points is a stable composition in view of Corollary 14. Finally, the Lawrence extensions are attached to the corresponding invisible regions such that an extended von Staudt polytope arises.

Having performed all the attachings (for all $j$ ), we are almost done. The space of possible construction is designated to be the union of realization spaces the Main Theorem concerns. We already know that the mapping of forgetting the attachings forms a stable composition. However, it still remains to answer the following questions:

(i) Does the face lattice of the constructed polytope only depend on the sign-vector $\left(\operatorname{sign}_{1}, \ldots, \operatorname{sign}_{m}\right)$ ?

(ii) Is the face lattice an injective function of the sign-vector?

(iii) Is the construction invertible? More precisely, this question reads as follows. Given a polytope in the union of the realization spaces of the possible constructions, which is now given by its lattice. Is such a realization necessarily the product of a construction as explained above?

If the latter questions can be answered affirmatively, then the Main Theorem is implied (except the assertion concerning computability). 
The base of the construction was the space of starting polytopes, which is a subspace of a realization space. This subspace is determined by means of a stable composition of the realization space to the space of ordered $k$-tuples. The latter stable composition is defined geometrically (mapping $\xi$ ). In fact, the space of starting polytopes constitutes the inverse images of $\mathcal{R}_{\mathcal{C}}$. It is partitioned by the inverse images of the spaces $\mathcal{R}_{\mathcal{C}}(\sigma)$. (This partition is stably equivalent to the partition of $(1, \infty)^{n}$ into basic semialgebraic sets treated in the Main Theorem.)

By an inductive process, polytopes have been attached to specific facets. In all steps the face lattice of the resulting polytope collected all the faces of the original polytope and the attached polytope with one exception, namely the facet of attaching. This procedure is called the connected sum operation; see [9]. The face lattices of the attached polytopes are the same in the following cases (for fixed $j$ ):

- $P^{1}$

- $P^{2}$ in the cases where a condition has been treated,

- $P_{0}^{2}$,

- von Staudt polytopes,

- Lawrence extensions.

The face lattices of the attached polytopes $\left[P^{1}, P_{0}^{2}, P^{2}\right]$ are the same for all cases where the image of the corresponding sign-function coincides. Different values of the signfunction produced different face lattices. This is implied by Corollary 11 . Hence questions (i) and (ii) are answered affirmatively.

For question (iii) we recall that the construction process never deletes a face of dimension 2 or less. This implies that the 2-skeleton of any facet disappearing from the face lattice (by the attaching process) remains present. However, the removed facet was the convex hull of a pair of bundled polygons. The bundling is a property of the 2-skeleton, therefore the convex hull of the 2-skeleton belonging to a removed facet will be threedimensional in all realizations of the resulting family of polytopes (see Lemma 10). This property has also been used in the work of Richter-Gebert [9], called necessary flatness. The latter argumentation implies that the construction can be inverted step by step. We show that the realization of the starting polytope obtained by deconstruction is a starting polytope. In fact, the property of the von Staudt polytope to encode a projective isomorphism, in connection with the property of bundled encoding polygons to express the order type by means of the face lattice of their convex hull, also shows this.

It remains to verify the complexity result. Indeed, both numbers $k$ and $l$ are $O(\alpha)$. From the (bundled) product structure of the starting polytope it then follows that its face lattice (including the order structure) is $O\left(\alpha^{2}\right)$. (There are faces with $O(\alpha)$ sublattice, however, there are only $O(\alpha)$ of them.) Moreover, there are $O(\alpha)$ many attachings. The face lattice of each attached polytope can be estimated by $O(\alpha)$. Altogether, each lattice $\mathcal{L}_{\sigma}$ is $O\left(\alpha^{2}\right)$, and it can also be computed within this time (including all the intermediate steps).

A modified construction provides an incidence code of complexity $O(\alpha)$. The difference to the described construction is a restriction of the number of copies of the original $(k+3)$-gon to $O(1)$. In fact, one starts with a bundled triple of encoding $(k+3)$-gons, say $\left[P^{a}, P^{b}, P^{d}\right]$. Put $P^{c}:=P^{d}$. Then the hexagons $P^{1}, \widetilde{P}^{2}, P^{2}$, and the (extended) von Staudt polytope are attached as described, but to the facet $\left[P^{c}, P^{d}\right]$. After each step 
of fixing one condition or sign-function the face $\widetilde{P}^{2}$ (or $P^{2}$ ) is denoted by $P^{c}$, and the inductive process proceeds.

Remark 16. The number of vertices of the lattices $\mathcal{L}_{\sigma}$ can be bounded by $2{ }^{10} \alpha$.

\section{The Face Lattice of the von Staudt Polytope}

Since $\left(q^{1}, q^{2}\right)$ is an ordered 6-tuple, Lemma 10 determines the lattice of $\left[P^{1}, P^{2}\right]$. By the construction, we have

$$
\begin{aligned}
& \operatorname{proj}\left(p_{\infty}, p_{4}^{1}\right) \cap \operatorname{proj}\left(p_{2}^{1}, p_{3}^{1}\right)=\operatorname{proj}\left(p_{\infty}, p_{4}^{1}\right) \cap \operatorname{proj}\left(p_{2}^{3}, p_{3}^{3}\right), \\
& \operatorname{proj}\left(p_{\infty}, p_{4}^{1}\right) \cap \operatorname{proj}\left(p_{1}^{1}, p_{2}^{1}\right)=\operatorname{proj}\left(p_{\infty}, p_{4}^{1}\right) \cap \operatorname{proj}\left(q_{1}^{\prime}, p_{2}^{3}\right) .
\end{aligned}
$$

This, in analogy to Lemma 10 , determines the face lattice of $\left[P^{1}, P^{3}\right]$. Analogously, we know the face lattice of $\left[P^{2}, P^{4}\right]$. In a similar way, the face lattice of $\left[P^{3}, P^{4}\right]$ is given: by construction we have

$$
\begin{aligned}
& \operatorname{proj}\left(p_{\infty}, q_{1}^{\prime}\right) \cap \operatorname{proj}\left(p_{2}^{3}, p_{3}^{3}\right)=\operatorname{proj}\left(p_{\infty}, q_{1}^{\prime}\right) \cap \operatorname{proj}\left(p_{2}^{4}, p_{3}^{4}\right)=q_{2}^{\prime}, \\
& \operatorname{proj}\left(p_{\infty}, q_{1}^{\prime}\right) \cap \operatorname{proj}\left(p_{3}^{3}, p_{4}^{1}\right)=\operatorname{proj}\left(p_{\infty}, q_{1}^{\prime}\right) \cap \operatorname{proj}\left(p_{3}^{4}, p_{4}^{2}\right)=q_{3}^{\prime} .
\end{aligned}
$$

Figure 15 shows a Schlegel diagram of our construction. We emphasize the already known part of the face lattice, which is also shown in the smaller subfigures. We look inside the von Staudt polytope through its facet $\left[P^{1}, P^{2}\right]$; thus $\left[P^{1}, P^{2}\right]$ forms the boundary of the three-dimensional figure.

To complete our proof, we explicitly list the remaining facets of the von Staudt polytope $P$. We use the fact that any 2 -face of a 4-polytope belongs to exactly two facets. So far we have found four facets (and their 2-faces). From now on they are called known facets (or faces). A known 2-face that is contained in two of the known facets is referred to as used, the other ones (which merely belong to one known facet) are called unused. At this stage of the analysis, the only used 2-faces are $P^{1}, P^{2}, P^{3}$, and $P^{4}$.

Any vertex is contained in at least one already known facet. This for topological reasons implies that a vertex that belongs to a new (still unknown) facet is contained in an unused 2-face. In particular, $p_{\infty}$ cannot belong to any new facet. In the remainder of the proof we find the still unknown facets one by one. After each step we change our sets of known facets (faces) and of the (un-) used 2-faces. Each step starts from an unused 2-face and finds the unknown facet containing it.

Facet $F_{1}$. We are searching for the still unknown facet containing the triangular face $\left(p_{4}^{1}, p_{4}^{2}, p_{3}^{2}\right)$, which is known as a face of $\left[P^{1}, P^{2}\right]$. Since $F_{1}$ differs from $\left[P^{1}, P^{2}\right]$, no other vertex of $F_{1}$ may be from this facet. The other vertices of $F_{1}$ are thus from $P^{3}$ and $P^{4}$, excluding $p_{\infty}$. If $p_{2}^{3}$ would be vertex of $F_{1}$, then by $p_{4}^{1} \in F_{1}$ we would have $P^{1} \subset F^{1}$, which contradicts $p_{\infty} \notin F_{1}$. Analogously, we see that $p_{2}^{4}, q_{1}^{\prime} \notin F_{1}$. The only candidates for vertices of $F_{1}$ are thus $p_{3}^{3}$ and $p_{3}^{4}$. However, both points, together with $p_{4}^{1}$ and $p_{4}^{2}$, form the vertex vector of a 2 -face (of $\left[P^{3}, P^{4}\right]$ ). Hence either both or none of them belongs to $F_{1}$, and thus in fact both. This describes the vertex vector of $F_{1}$. We see 

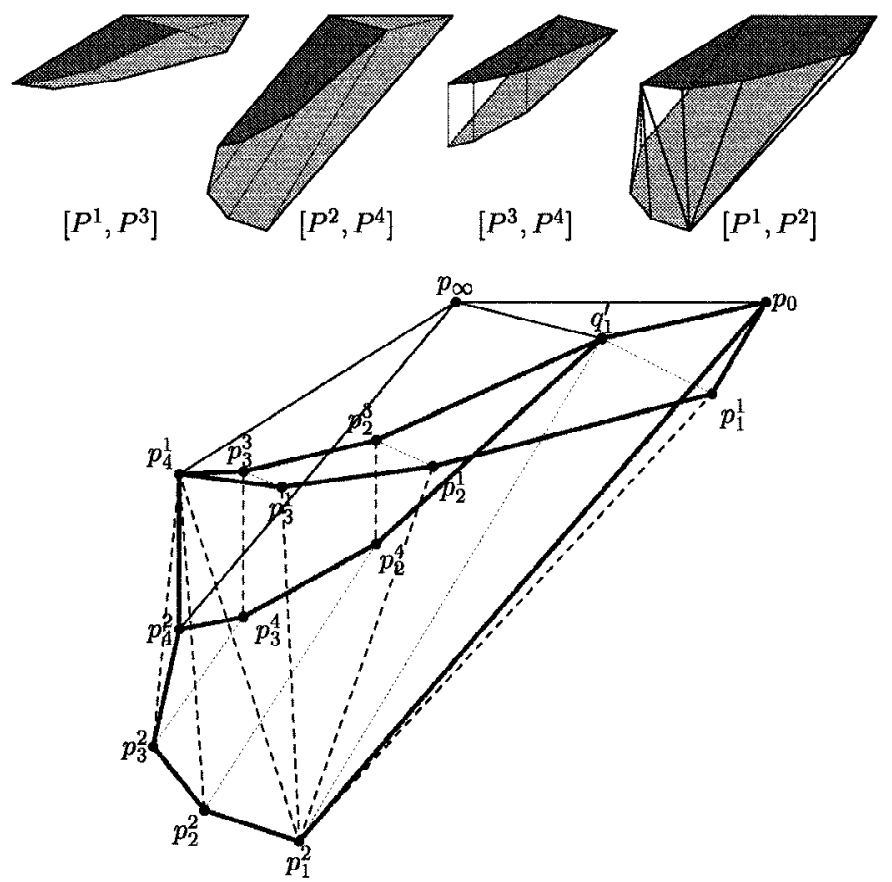

Fig. 15. The von Staudt polytope: the known facets.

that $F_{1}$ is the pyramid over a quadrilateral face of $P^{3}, P^{4}$. This provides the face lattice of $F_{1}$; see Fig. 16.

Note that two unused 2-faces became used, whereas two new unused 2-faces arose. Moreover, from now on, vertex $p_{4}^{2}$ cannot belong to any new facet. The vertices of $P$ that are excluded as vertices of new facets, are marked by unfilled dots. The shaded areas in Fig. 16 show the yet unused 2 -faces that are not from the obvious facets $\left[P^{1}, P^{2}\right]$, $\left[P^{3}, P^{4}\right],\left[P^{1}, P^{3}\right]$, or $\left[P^{2}, P^{4}\right]$. For easier visual checking we have outlined the union of the unused 2-faces belonging to $\left[P^{1}, P^{3}\right]$ and $\left[P^{2}, P^{4}\right]$.

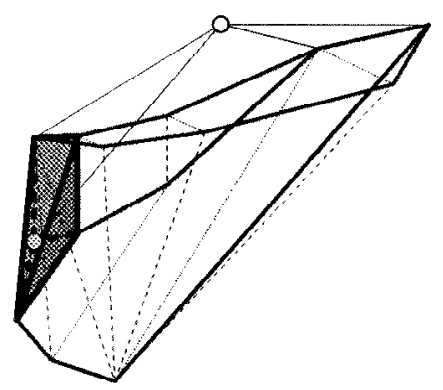

Fig. 16. Facet $F_{1}$. 


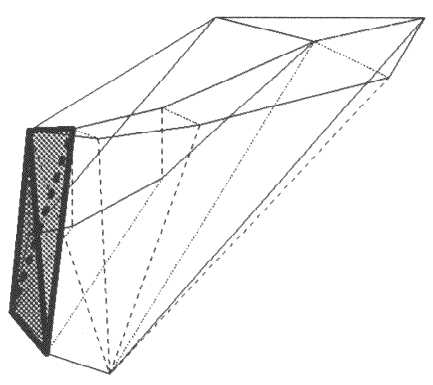

Fig. 17. Facet $F_{2}$.

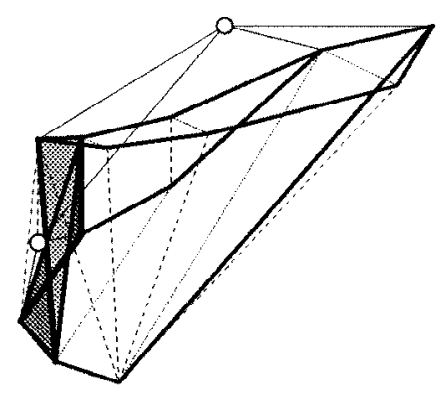

Fig. 18. Where is $F_{3}$ ?

Facet $F_{2}$. This time we start with the unused 2-face $\left(p_{4}^{1}, p_{3}^{2}, p_{2}^{2}\right) \subset\left[P^{1}, P^{2}\right]$. The remaining vertices of $F_{2}$ come from $P^{3}$ and $P^{4}$, as above. The open line segment $\left(p_{4}^{1}, p_{2}^{4}\right)$ lies in the relative interior of $\left[P^{3}, P^{4}\right]$; hence $p_{2}^{4} \notin F_{2}$. This, with respect to the quadrilateral face $\left(p_{3}^{2}, p_{2}^{2}, p_{2}^{4}, p_{3}^{4}\right)$, implies that $p_{3}^{4}$ is not in $F_{2}$ either. The only remaining candidate is $p_{3}^{3}$ (other vertices of $P^{3}$ are forbidden by $p_{4}^{1} \in P^{3}$ ), so we take it. In the Figs. 17 and 18 we show the new facet $F_{2}$, and the situation of unused faces (not from the obvious facets) after $F_{2}$ became known.

Facet $F_{3}$. We start with $\left(p_{3}^{2}, p_{3}^{3}, p_{3}^{4}\right) \in \mathcal{L}\left(F_{1}\right)$. Since $F_{3} \neq F_{1}$, we have $p_{4}^{1} \notin F_{3}$. The open line segments connecting $p_{3}^{2}$ with points $p_{3}^{1}, p_{2}^{1}, p_{1}^{1}, p_{0},\left(q_{1}^{\prime}\right)$ respectively, lie within the relative interior of facet $\left[P^{1}, P^{2}\right],\left(\left[P^{2}, P^{4}\right]\right)$. This excludes the latter points from $F_{3}$. The only remaining candidates are $p_{2}^{2}, p_{2}^{3}$, and $p_{2}^{4}$, but none of these points can become a vertex of $F_{3}$ without the other ones (result of quadrilateral faces, as above). Therefore, $F_{3}=\left(p_{3}^{2}, p_{3}^{3}, p_{3}^{4}, p_{2}^{2}, p_{2}^{3}, p_{2}^{4}\right)$. Since $\left(p_{3}^{2}, p_{2}^{2}, p_{3}^{3}\right)$ is already known as a face of $P$, the face lattice of $F_{3}$ is uniquely determined. (Note that this verification requires knowledge of $F_{2}$ !) From now on, $p_{3}^{4}$ and $p_{3}^{2}$ are omitted from the remaining facets. In Figs. 19 and 20 we show the new facet $F_{3}$, and the situation of unused faces (not from the obvious facets) after $F_{3}$ became known.

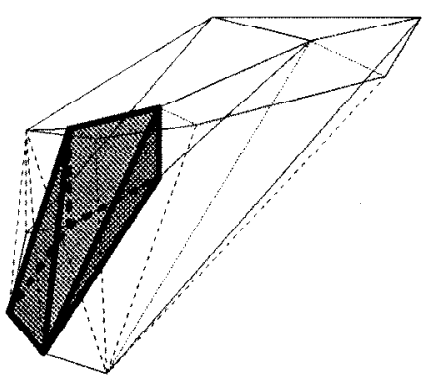

Fig. 19. Facet $F_{3}$.

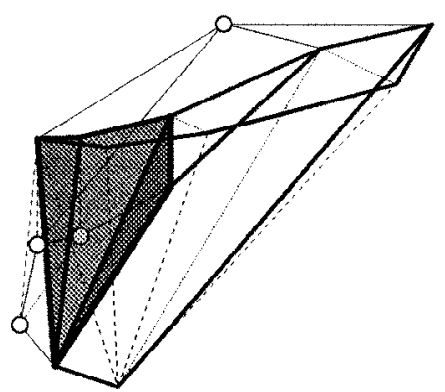

Fig. 20. Where is $F_{4}$ ? 


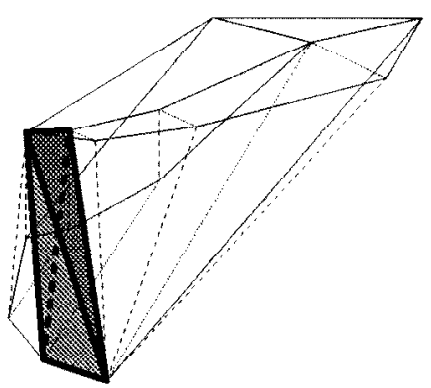

Fig. 21. Facet $F_{4}$.

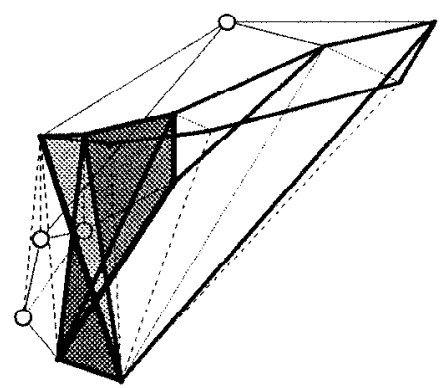

Fig. 22. Where is $F_{5}$ ?

Facet $F_{4}$. Start with $\left(p_{2}^{2}, p_{1}^{2}, p_{4}^{1}\right)$, which implies that $F_{4}$ has no other vertex from $\left[P^{1}, P^{2}\right]$. From $p_{4}^{1}$ we see that the only possible vertex from $\left[P^{3}, P^{4}\right]$ is $p_{3}^{3}$, thus $F_{4}=$ $\left(p_{2}^{2}, p_{1}^{2}, p_{4}^{1}, p_{3}^{3}\right) .\left(F_{4}\right.$ is shown in Fig. 21 , and in Fig. 22 we have the new situation.)

Facet $F_{5}$. Start with $\left(p_{4}^{1}, p_{3}^{1}, p_{1}^{2}\right)$; then the other vertices of $\left[P^{1}, P^{2}\right]$ are excluded. As above, from $p_{4}^{1}$, it follows that only $p_{3}^{3}$ remains as a candidate; i.e., $F_{5}=\left(p_{4}^{1}, p_{3}^{1}, p_{1}^{2}, p_{3}^{3}\right)$. Now $p_{4}^{1}$ disappears from the list of possible vertices of new facets. ( $F_{5}$ is shown in Fig. 23 , and in Fig. 24 we have the new situation.)

Facet $F_{6}$. This time we start with $\left(p_{1}^{2}, p_{2}^{2}, p_{3}^{3}\right)$. The open line segments connecting $p_{2}^{2}$ with the points $p_{3}^{1}, p_{2}^{1}, p_{1}^{1}$, and $p_{0}$ are contained in the relative interior of $\left[P^{1}, P^{2}\right]$, hence $F_{6}$ does not intersect $P^{1}$. We know that $q_{1}^{\prime} \notin F_{6}$, since otherwise $P^{3} \subset F_{6}$. From $F_{6} \neq F_{3}$ it follows that $p_{2}^{4} \notin F_{6}$. The only remaining candidate for a vertex of $F_{6}$ is therefore $p_{2}^{3}$. ( $F_{6}$ is shown in Fig. 25, and in Fig. 26 we have the new situation.)

Facet $F_{7}$. Start with $\left(p_{2}^{2}, p_{2}^{3}, p_{2}^{4}\right)$. The vertices of $P^{1}$ are excluded for the same reason as in $F_{6}$, whereas $p_{3}^{3}$ is omitted by $F_{7} \neq F_{3}$. As candidates there remain $p_{1}^{2}$ and $q_{1}^{\prime}$, but in view of the quadrilateral face $\left(p_{2}^{4}, p_{2}^{2}, p_{1}^{2}, q_{1}^{\prime}\right)$ their appearance as vertices of $F_{7}$ is equivalent. Therefore $F_{7}=\left(p_{2}^{2}, p_{2}^{3}, p_{2}^{4}, p_{1}^{2}, q_{1}^{\prime}\right)$. Since $\left(p_{2}^{2}, p_{1}^{2}, p_{2}^{3}\right)$ is already known as

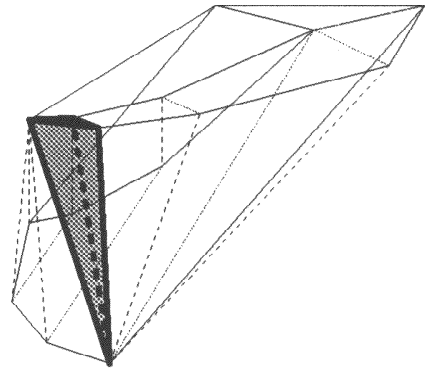

Fig. 23. Facet $F_{5}$.

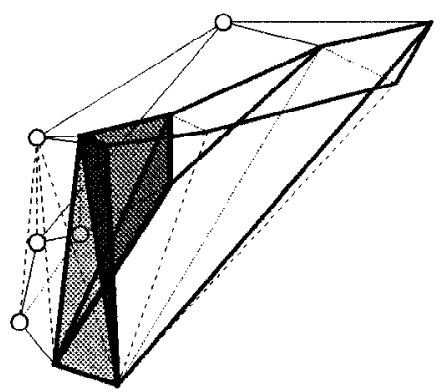

Fig. 24. Where is $F_{6}$ ? 


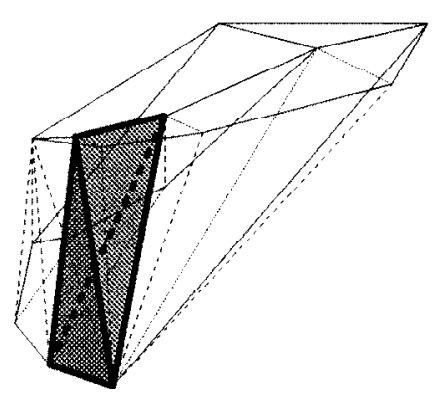

Fig. 25. Facet $F_{6}$.

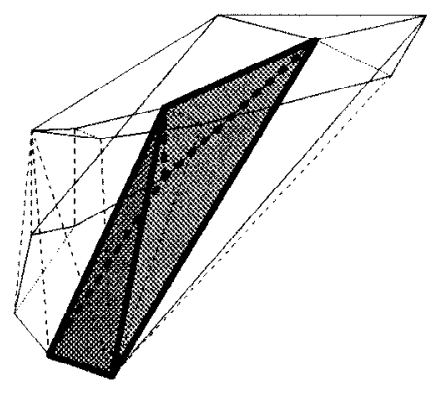

Fig. 27. Facet $F_{7}$.

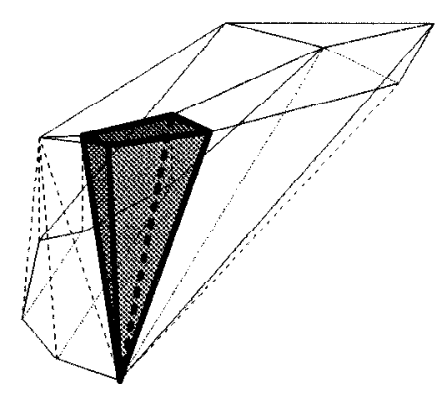

Fig. 29. Facet $F_{8}$.

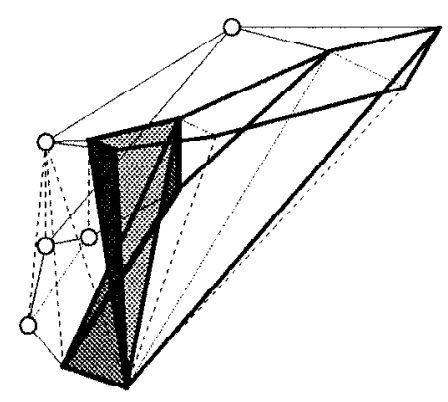

Fig. 26. Where is $F_{7}$ ?

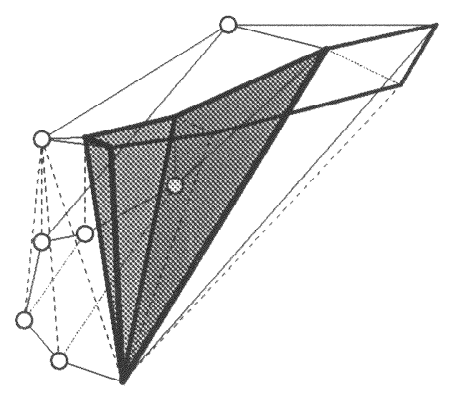

Fig. 28. Where are $F_{8} \ldots$

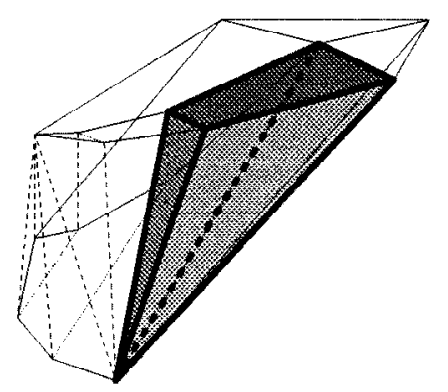

Fig. 30. Facet $F_{9}$. 


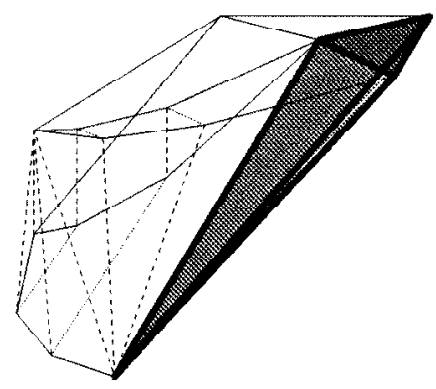

Fig. 31. Facet $F_{10}$.

a face of $P$, the face lattice of $F_{7}$ is uniquely determined. ( $F_{7}$ is shown in Fig. 27, and in Fig. 28 we have the new situation.)

Facets $F_{8}-F_{10}$. The only vertices of $P$ that are vertices of still unknown facets, are $p_{3}^{1}, p_{2}^{1}, p_{1}^{1}, p_{0}, q_{1}^{\prime}, p_{2}^{3}, p_{3}^{3} \in\left[P^{1}, P^{3}\right]$ and $p_{1}^{2}$. Therefore, the remaining facets of $P$ are pyramids (with the top vertex $p_{1}^{1}$ ) over the unused 2 -faces of $\left[P^{1}, P^{3}\right]$, i.e., we have $F_{8}=\left(p_{3}^{1}, p_{2}^{1}, p_{2}^{3}, p_{3}^{3}, p_{1}^{2}\right), F_{9}=\left(p_{2}^{1}, p_{1}^{1}, q_{1}^{\prime}, p_{2}^{3}, p_{1}^{2}\right)$, and $F_{10}=\left(p_{1}^{1}, p_{0}, q_{1}^{\prime}, p_{1}^{2}\right)$. The corresponding face lattices are obvious. Now all 2-faces are used, i.e., the analysis of the face lattice $\mathcal{L}(P)$ is completed. The facets $F_{8}-F_{10}$ are shown in Figs. 29-31.

\section{Acknowledgments}

I would like to thank Bert Jongen for his continuing support. Moreover, the remarks by two anonymous referees, concerning both mathematical precision and stylistic aspects, are gratefully acknowledged.

\section{References}

1. Bochnak, J., Coste, M., and Roy, M.-F., Géométrie Algébrique Réelle, Springer-Verlag, Berlin, 1987.

2. Bokowski, J., and Sturmfels, B., Computational Synthetic Geometry, Lecture Notes in Mathematics, Vol. 1355, Springer-Verlag, Berlin, 1989.

3. Grünbaum, B., Convex Polytopes, Interscience, London, 1967.

4. Günzel, H., The Universal Partition Theorem for Oriented Matroids, Discrete \& Computational Geometry, 15 (1996), 121-145.

5. Günzel, H., Hirabayashi, R., and Jongen, H. Th., Multiparametric Optimization: on Stable Singularities Occurring in Combinatorial Partition Codes, Control \& Cybernetics, 23 (1994), 153-167.

6. Hilbert, D., Grundlagen der Geometrie, 2nd edition, Teubner, Leipzig, 1903.

7. Mnëv, N.E., The Universality Theorems on the Classification Problem of Configuration Varieties and Convex Polytopes Varieties, in: Topology and Geometry-Rohlin Seminar, Viro, O.Y., ed., pp. 527-543, Lecture Notes in Mathematics, Vol. 1346, Springer-Verlag, Berlin, 1988.

8. Mnëv, N.E. The Universality Theorem on the Oriented Matroid Stratification of the Space of Real Matrices, DIMACS Series in Discrete Mathematics and Theoretical Computer Science, Vol. 6, American Mathematical Society, Providence, RI, 1991. 
9. Richter-Gebert, J., Realization Spaces of Polytopes, Lecture Notes in Mathematics, Vol. 1643, SpringerVerlag, Berlin, 1996.

10. Richter-Gebert, J., and Ziegler, G., Realization Spaces of 4-Polytopes Are Universal, Bulletin of the American Mathematical Society, 32 (1995), 403-412.

11. Shor, P., Stretchability of Pseudolines is NP-hard (The Victor Klee Festschrift), DIMACS Series in Discrete Mathematics and Theoretical Computer Science, Vol. 4, American Mathematical Society, Providence, RI, 1991.

12. Steinitz, E., Polyeder und Raumteilungen, Encyclopädie der Mathematischen Wissenschaften, Band 3 (Geometrie), Teil 3AB12, pp. 1-139, Teubner, Leipzig. 1922.

13. Young, J.W., Projective Geometry, Open Court, Chicago, 1930.

14. Ziegler, G.M., Lectures on Polytopes, Springer-Verlag, New York, 1995.

Received December 19, 1995, and in revised form December 16, 1996, April 28, 1997, and September 10, 1997. 